Carbon fragmentation measurements and validation of the Geant4 nuclear reaction models for hadrontherapy

This article has been downloaded from IOPscience. Please scroll down to see the full text article. 2012 Phys. Med. Biol. 577651

(http://iopscience.iop.org/0031-9155/57/22/7651)

View the table of contents for this issue, or go to the journal homepage for more

Download details:

IP Address: 193.205.228.106

The article was downloaded on 16/09/2013 at 15:17

Please note that terms and conditions apply. 


\title{
Carbon fragmentation measurements and validation of the GEANT4 nuclear reaction models for hadrontherapy
}

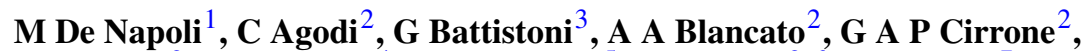 \\ G Cuttone $^{2}$, F Giacoppo ${ }^{4}$, M C Morone ${ }^{5}$, D Nicolosi ${ }^{2,6}$, L Pandola $^{7}$,

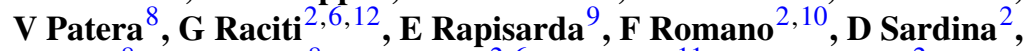

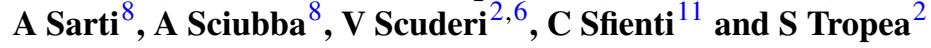 \\ ${ }^{1}$ INFN-Sezione di Catania, 64 Via S Sofia, I-95123 Catania, Italy \\ 2 INFN-Laboratori Nazionali del Sud, 62 Via S Sofia, I-95123 Catania, Italy \\ 3 INFN-Sezione di Milano, 16 Via Celoria I-20133 Milano, Italy \\ ${ }^{4}$ Department of Physics, University of Oslo, PO Box 1048 Blindern N-0316 Oslo, Norway \\ ${ }^{5}$ Dipartimento di Biomedicina e Prevenzione, Università di Roma Tor Vergata and Sezione INFN \\ di Roma Tor Vergata, Rome, Italy \\ ${ }^{6}$ Dipartimento di Fisica e Astronomia, Università degli Studi di Catania, 64 Via S Sofia, \\ I-95123 Catania, Italy \\ ${ }^{7}$ INFN-Laboratori Nazionali del Gran Sasso, SS 17 bis km 18+910, I-67100 Assergi (AQ), Italy \\ ${ }^{8}$ Dipartimento di Scienze di Base e Applicate per l'Ingegneria, La Sapienza Università di Roma, \\ Italy \\ ${ }^{9}$ Nuclear and Radiation Physics Section, Katholieke Universiteit Leuven, Celestijnenlaan 200d, \\ 24183001 Heverlee, Belgium \\ ${ }^{10}$ Museo Storico della Fisica e Centro Studi e Ricerche 'E Fermi', Compendio del Viminale, \\ Piazza del Viminale 1, 00184 Rome, Italy \\ ${ }^{11}$ Institut für Kernphysik Johannes Gutenberg, Universität Mainz Johann, \\ Joachim-Becher Weg 45, D-55099 Mainz, Germany \\ E-mail: marzio.denapoli@ct.infn.it
}

Received 9 June 2012, in final form 25 September 2012

Published 2 November 2012

Online at stacks.iop.org/PMB/57/7651

\begin{abstract}
Nuclear fragmentation measurements are necessary when using heavy-ion beams in hadrontherapy to predict the effects of the ion nuclear interactions within the human body. Moreover, they are also fundamental to validate and improve the Monte Carlo codes for their use in planning tumor treatments. Nowadays, a very limited set of carbon fragmentation cross sections are being measured, and in particular, to our knowledge, no double-differential fragmentation cross sections at intermediate energies are available in the literature. In this work, we have measured the double-differential cross sections and the angular distributions of the secondary fragments produced in the ${ }^{12} \mathrm{C}$ fragmentation at $62 \mathrm{~A} \mathrm{MeV}$ on a thin carbon target. The experimental data have been used to benchmark the prediction capability of the GEANT4 Monte Carlo code at intermediate energies, where it was never tested before. In particular,
\end{abstract}

${ }^{12}$ Deceased. 
we have compared the experimental data with the predictions of two GEANT4 nuclear reaction models: the Binary Light Ions Cascade and the Quantum Molecular Dynamic. From the comparison, it has been observed that the Binary Light Ions Cascade approximates the angular distributions of the fragment production cross sections better than the Quantum Molecular Dynamic model. However, the discrepancies observed between the experimental data and the Monte Carlo simulations lead to the conclusion that the prediction capability of both models needs to be improved at intermediate energies.

(Some figures may appear in colour only in the online journal)

\section{Introduction}

The use of heavy-ion beams in hadrontherapy is motivated by the highly localized dose distribution that they provide at the end of the radiation range, i.e. the Bragg peak, and by the enhanced relative biological effectiveness with respect to photon or proton irradiation. Thanks to these advantages, carbon beams are currently used in some hadrontherapy facilities around the world (Amaldi and Kraft 2005, Schardt et al 2010). However, as carbon nuclei penetrate the human tissues, they may undergo inelastic nuclear reactions leading to the production of secondary fragments. Such fragments have different ranges and angular distributions with respect to the primary ions, and thus, the resulting spatial dose distribution, inside and outside the tumor region, differs from that of the primary beam. Moreover, the different linear energy transfer (LET) of the secondary ions with respect to the carbon beam results in a different biological effectiveness for the same delivered dose. Therefore, all these effects arising from the carbon fragmentation have to be correctly evaluated when planning a tumor treatment. Treatment planning systems are currently based on relatively fast deterministic codes (Krämer and Durante 2010, Sihver and Mancusi 2009). Nevertheless, the only way to overcome the shortcomings of analytical calculations in the case of mixed radiation fields and complex geometries is the use of reliable Monte Carlo codes, such as GeAnT4 (Agostinelli et al 2003, Allison et al 2006), FLUKA (Battistoni et al 2007, Fassò et al 2005), MCNPX (Hughes et al 1997 , LANL 2002), SHIELD/SHIELD-HIT (Dementyev and Sobolevsky 1999, Gudowska et al 2004) and PHITS (Niita et al 2006). However, in order to precisely simulate the spatial dose distribution and the resulting biological effects in the human body, the physical models used in the Monte Carlo codes need to be tuned and validated by experimental fragmentation data.

The ability of FLUKA and GEANT4 to reproduce carbon fragmentation data has been recently investigated in Böhlen et al (2010) by using experimental data obtained with carbon beams impinging on thick water and polycarbonate targets in the energy range of 100-500 A MeV. The comparison between the nuclear reaction model predictions and the experimental data has shown discrepancies of the order of $10 \%$, at least (Böhlen et al 2010). These results suggest the need to improve the tested codes for their application to hadrontherapy. However, since a limited set of experimental fragmentation cross sections is available, and in particular, to our knowledge, no double-differential fragmentation cross sections at intermediate energies are present in the literature, a systematic of high-quality double-differential cross-section measurements is mandatory.

In order to provide an extensive set of fragmentation data for their use in hadrontherapy, we have studied the fragmentation of a ${ }^{12} \mathrm{C}$ beam at $62 \mathrm{~A} \mathrm{MeV}$ on a thin carbon target at the INFN-Laboratori Nazionali del Sud (LNS), Catania. The double-differential cross 


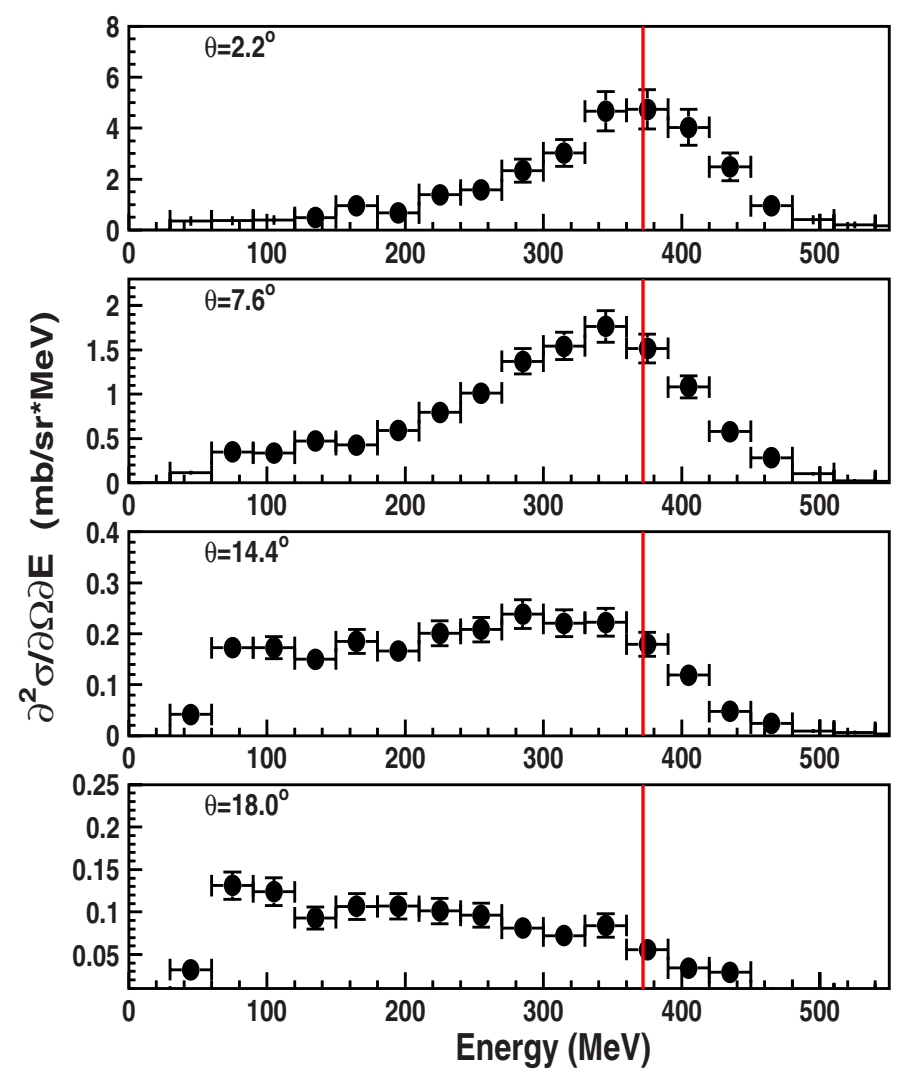

Figure 1. ${ }^{6} \mathrm{Li}$ double-differential cross sections measured at four angles $\left(\theta_{\text {lab }}=2.2^{\circ}, 7.6^{\circ}, 14.4^{\circ}\right.$ and $18^{\circ}$ ). The energy equivalent to the beam velocity is indicated by a solid line.

sections and the angular distributions of the secondary fragments have been measured over a wide angular range. The availability of double-differential fragmentation cross sections at intermediate energy is of particular interest in order to accurately predict the fluences of the secondary fragments and their angular distributions within the human tissues. Indeed, although therapeutic carbon beams have energies of the order of hundreds of $\mathrm{A} \mathrm{MeV}$, the carbon ions lose their energy passing through the patient's body so that the inelastic nuclear reactions may occur at energies much lower than the incident ones.

Moreover, fragmentation measurements performed with thin targets are of particular interest in benchmarking the nuclear interaction codes, since in these measurements the fragmentation of the secondary products and the electromagnetic physics effects in the target are not relevant. Our measurements offer, therefore, a unique opportunity to test the performances of the nuclear reaction models implemented in the Monte Carlo codes for their use in hadrontherapy.

In this paper, we discuss the results of the comparison between the measured fragmentation cross sections and the GEANT4 predictions. In particular, two GEANT4 nuclear reaction models, i.e. the Binary Light Ions Cascade and the Quantum Molecular Dynamic, have been compared and evaluated. Our results represent the first comparison of the GEANT4 nuclear reaction models performed so far by using experimental data obtained with a thin target at intermediate energies. 


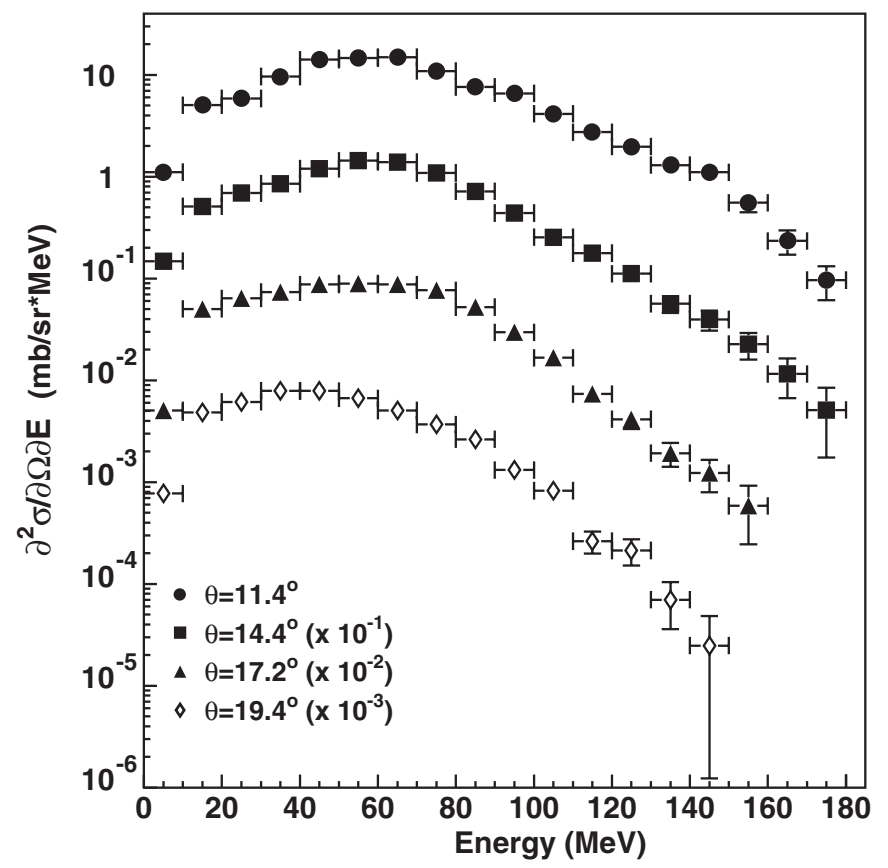

Figure 2. Double-differential cross sections of protons detected at four angles: $\theta_{\text {lab }}=11.4^{\circ}, 14.4^{\circ}$, $17.2^{\circ}$ and $19.4^{\circ}$. Cross sections at $\theta_{\text {lab }}=14.4^{\circ}, 17.2^{\circ}$ and $19.4^{\circ}$ are scaled by the factors $10^{-1}$, $10^{-2}$ and $10^{-3}$, respectively.

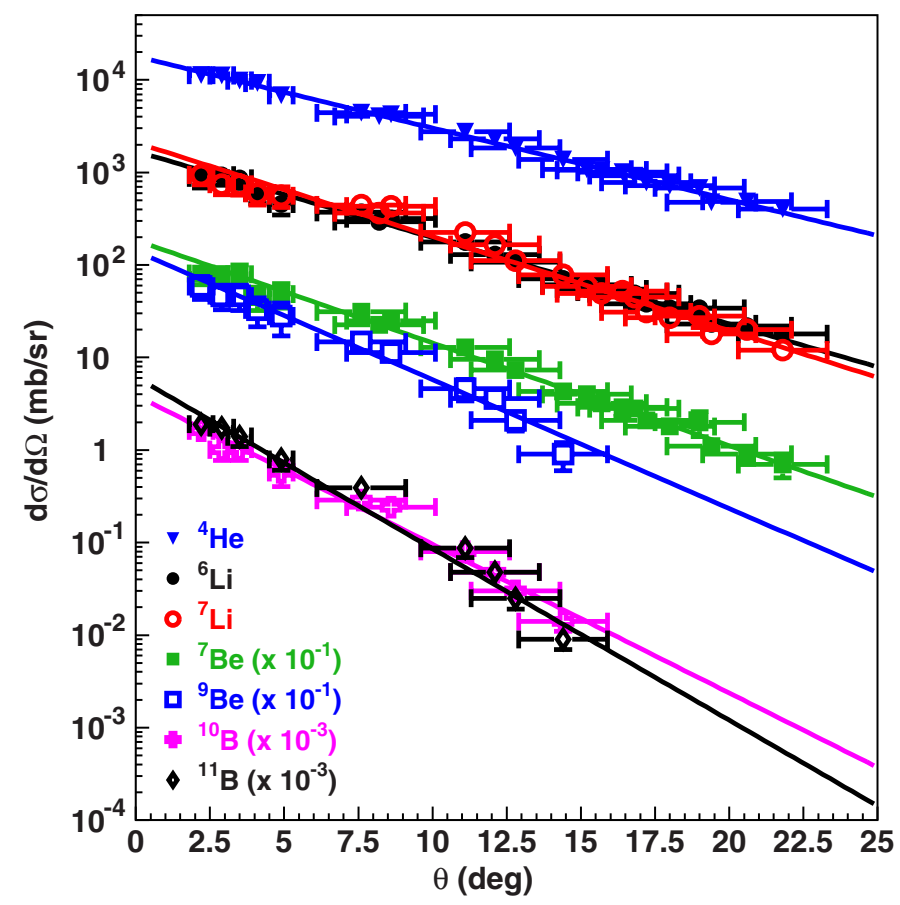

Figure 3. Angular distributions of the $Z>1$ fragments. The horizontal error bars represent the angular acceptance of the telescopes. Cross sections of Be and B isotopes have been scaled by factors of $10^{-1}$ and $10^{-3}$, respectively. The solid lines are exponential best-fits of the data. 


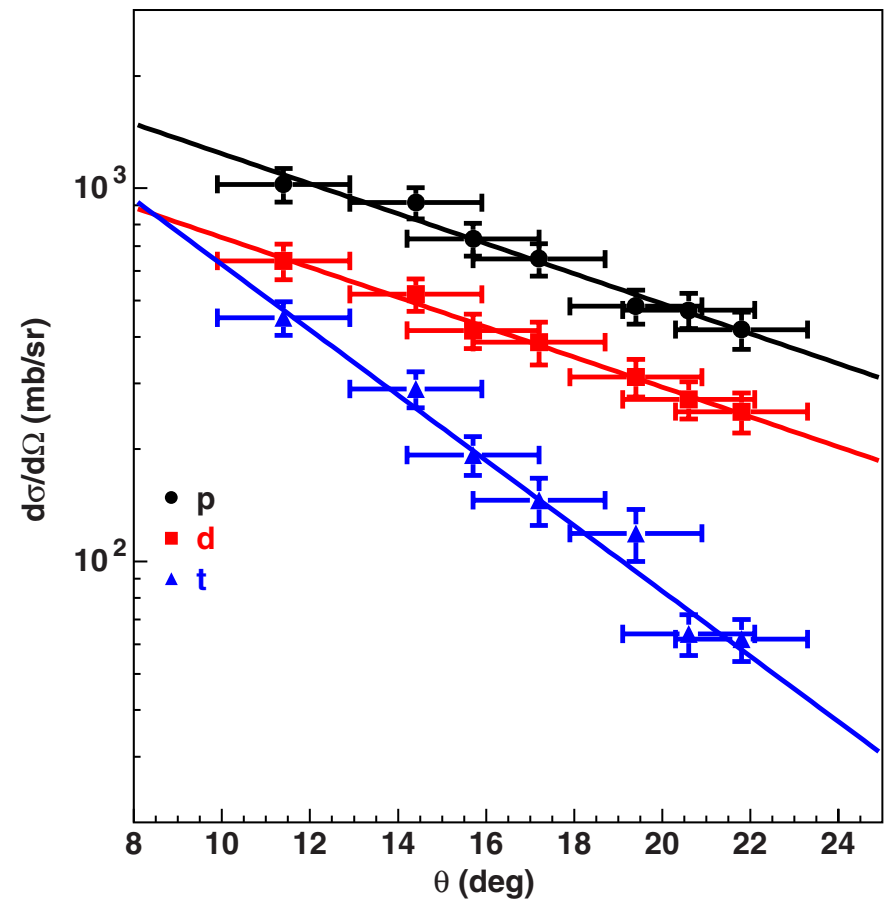

Figure 4. Angular distributions of the $Z=1$ isotopes. The horizontal error bars represent the angular acceptance of the telescopes. The solid lines are exponential best-fits of the data.

\section{The experimental setup}

The experiment was performed at the Superconducting Cyclotron (CS) of the INFN-LNS in Catania. A ${ }^{12} \mathrm{C}$ beam was accelerated at $62 \mathrm{~A} \mathrm{MeV}$ and sent to a $104 \mu \mathrm{g} \mathrm{cm}^{-2}$ carbon target. The energy and emission angle of charged fragments $(1 \leqslant Z \leqslant 5)$ produced in the interaction with the target were measured by a detection setup consisting of two Si-CsI hodoscopes (Raciti et al 2008):

- The Hodo-Small-formed by 81 twofold telescopes: $300 \mu \mathrm{m}$ silicon detectors $1 \times 1 \mathrm{~cm}^{2}$ of active area followed by a $1 \times 1 \mathrm{~cm}^{2}$ and $10 \mathrm{~cm}$ long $\mathrm{CsI}(\mathrm{Tl})$;

- The Hodo-Big-formed by 88 threefold telescopes: $50+300 \mu \mathrm{m}$ silicon detectors both having $3 \times 3 \mathrm{~cm}^{2}$ surface followed by a $6 \mathrm{~cm}$ long $\mathrm{CsI}(\mathrm{Tl})$.

In the current experiment, the Hodo-Small was placed $80 \mathrm{~cm}$ far from the target, covering the angular region $2.2^{\circ} \leqslant \theta_{\text {lab }} \leqslant 5.5^{\circ}$. The Hodo-Big was placed at $60 \mathrm{~cm}$ from the target covering the angular region $7.6^{\circ} \leqslant \theta_{\text {lab }} \leqslant 21.8^{\circ}$. Fragments detected in each telescope were identified in charge and mass by using the $\Delta E-E_{\text {res }}$ identification technique, with $\Delta E$ and $E_{\text {res }}$ being the energy loss and the residual energy measured in the Si and CsI detectors, respectively. The absolute cross sections were determined from the measured fragment yields, corrected for the acquisition system dead-time, the target thickness and the beam current measured with a Faraday cup. The uncertainties in the cross sections result from the statistical errors in the fragment yields, the uncertainties on the target thickness, the number of incident ions and the dead-time. 

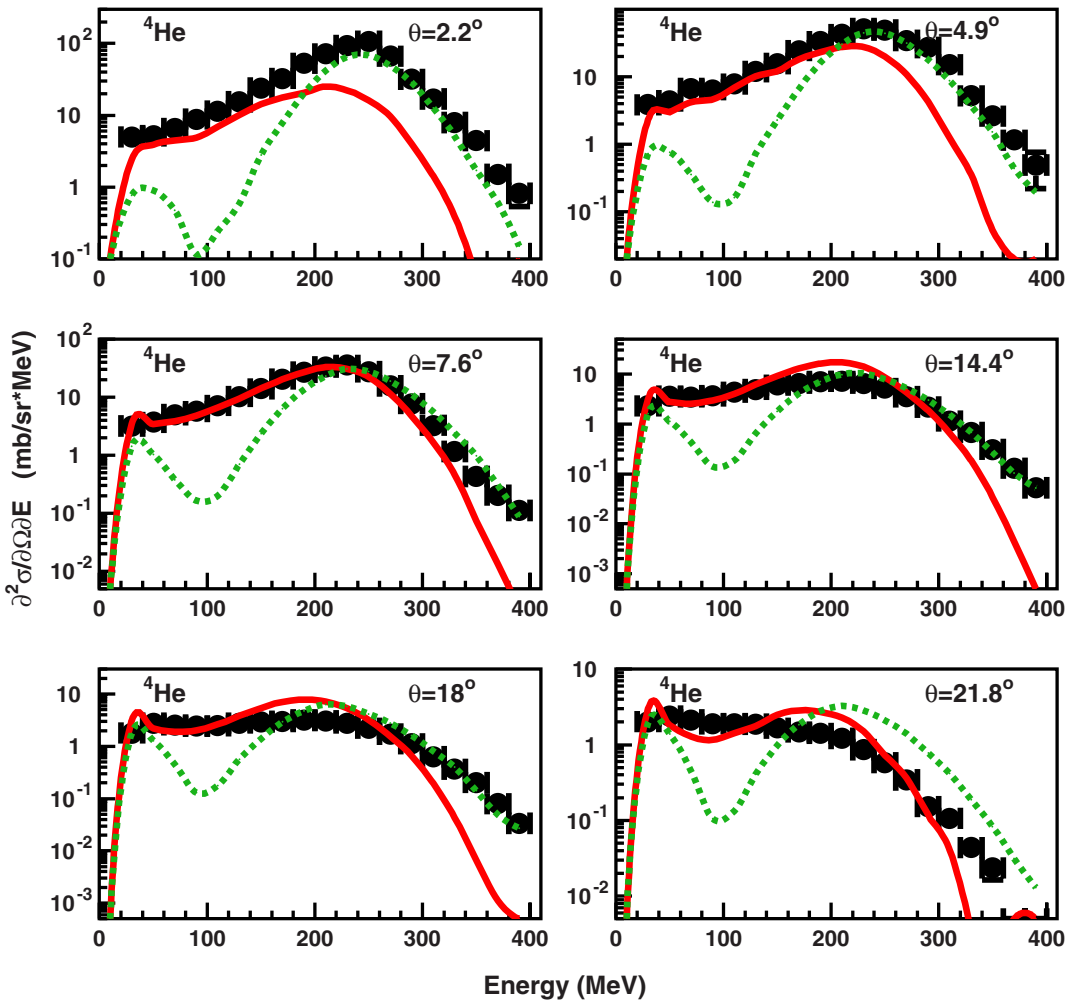

Figure 5. Double-differential cross sections measured at $\theta_{\mathrm{lab}}=2.2^{\circ}, 4.9^{\circ}, 7.6^{\circ}, 14.4^{\circ}, 18^{\circ}$ and $21.8^{\circ}$ for ${ }^{4} \mathrm{He}$ produced in the ${ }^{12} \mathrm{C}+{ }^{12} \mathrm{C}$ reaction at $62 \mathrm{~A} \mathrm{MeV}$. The data are compared with the Binary Light Ion Cascade (dashed lines) and the QMD (solid lines) predictions.

\section{Double-differential cross sections and angular distributions}

The measured double-differential production cross sections of the $Z>1$ fragments are listed in tables A1-A7 in the appendix. As an example, in figure 1, the ${ }^{6} \mathrm{Li}$ double-differential cross sections at four angles, $\theta_{\text {lab }}=2.2^{\circ}, 7.6^{\circ}, 14.4^{\circ}$ and $18^{\circ}$, are shown. As one can see from the figure, the energy distributions exhibit a Gaussian-like main peak, centered around the beam velocity (solid line) and a tail extending at lower energies. The main peak decreases and moves to lower energies as the emission angle increases. In contrast, the low-energy tail increases with the angle, becoming the dominant part of the energy spectrum at large angles. The energy distributions of all the fragments with $Z>1$ show a similar behavior. The presence of a Gaussian-like peak together with a low-energy tail in the fragment energy distributions has already been observed at intermediate energies (Dayras et al 1986, Glasow et al 1990, Tarasov et al 1998, Czudek et al 1991, Guet et al 1983, Pruneau et al 1989, Tarasov 2004). The Gaussian-like peak can be associated with those fragments produced in peripheral collisions. Indeed, these fragments are emitted at approximately the same velocity of the beam and in a relatively small angular cone around the beam direction. On the other hand, the fragments emitted at large angles and at velocities much lower than the beam are most probably produced in more central and dissipative collisions than the ones producing high-energy fragments.

The measured double-differential cross sections of the $Z=1$ isotopes are listed in tables A8-A10 in the appendix. Figure 2 shows, as an example, the proton double-differential 

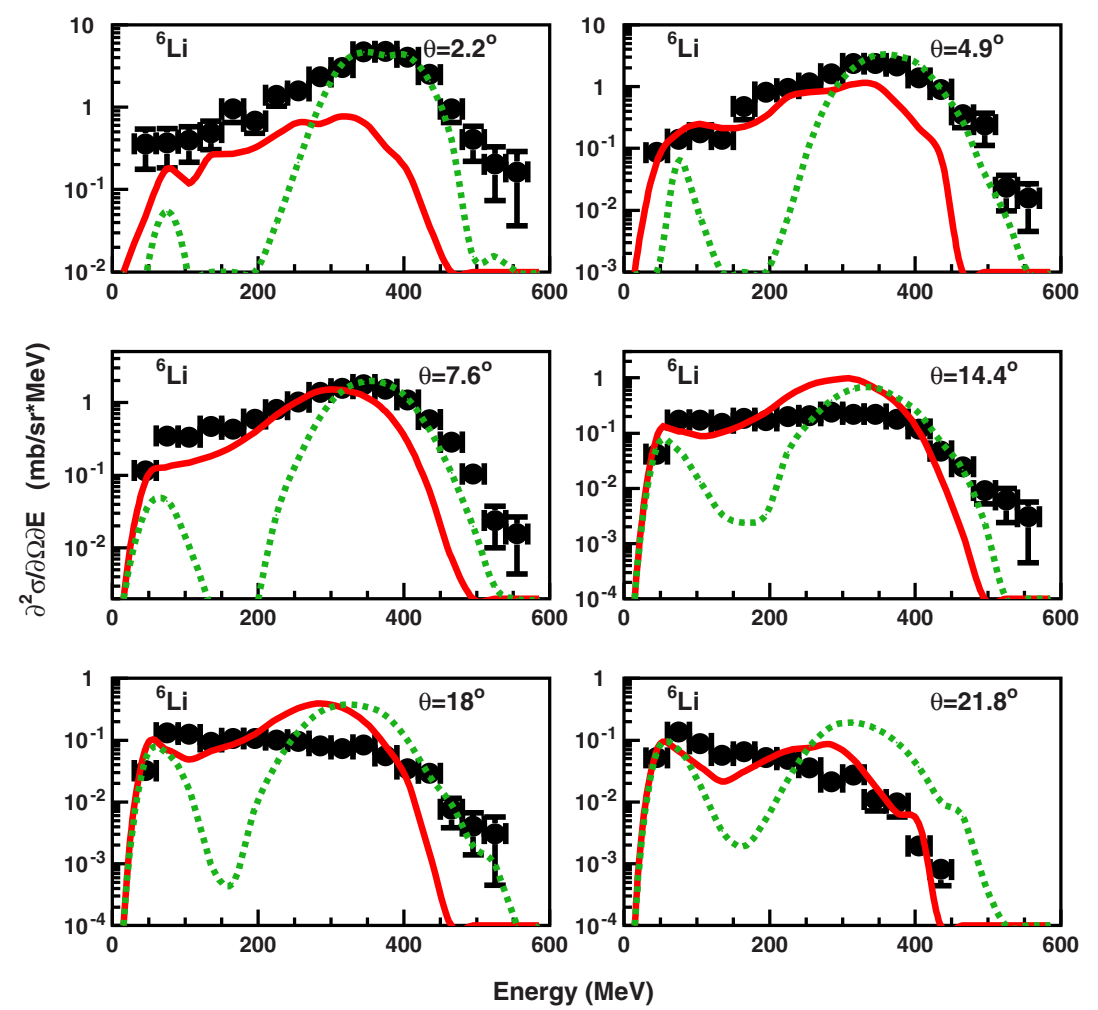

Figure 6. Double-differential cross sections measured at $\theta_{\mathrm{lab}}=2.2^{\circ}, 4.9^{\circ}, 7.6^{\circ}, 14.4^{\circ}, 18^{\circ}$ and $21.8^{\circ}$ for ${ }^{6} \mathrm{Li}$ produced in the ${ }^{12} \mathrm{C}+{ }^{12} \mathrm{C}$ reaction at $62 \mathrm{~A} \mathrm{MeV}$. The data are compared with the Binary Light Ion Cascade (dashed lines) and the QMD (solid lines) predictions.

cross sections at four angles: $\theta_{\mathrm{lab}}=11.4^{\circ}, 14.4^{\circ}, 17.2^{\circ}$ and $19.4^{\circ}$. As can be seen from the figure, the energy distributions fall off exponentially as the energy increases. A similar behavior has been also observed in other works where the $Z=1$ particles have been assumed to be isotropically emitted from one or more moving sources and their energy distributions have been commonly reproduced with a 'moving source fit' (see, e.g., Jacak et al 1987, Wada et al 1989).

The angular distributions of the secondary fragment production cross sections have been extracted by integrating in energy the double-differential cross sections at different angles. Figures 3 and 4 show the angular distributions of $Z>1$ and $Z=1$ fragments. The cross-section values, shown in figures 3 and 4, are listed in tables A11 and A12, respectively. The angular distributions of all isotopes have been fitted by using an exponential function; the results are shown in figures 3 and 4 as solid lines. As one can clearly see, the angular distributions fall exponentially, indicating that the secondary fragments are mostly emitted at forward angles. In particular, the slope of the angular distributions of heavy fragments is steeper than the one of light fragments indicating that the former are emitted in a narrower angular cone around the beam direction. Moreover, the angular distributions show unambiguously that the larger fraction of the produced fragments, in the explored angular region, are $\alpha$ particles. As is well known, because of their greater binding energy, $\alpha$ particles are very stable nuclei with respect to other light ones. This aspect may lead to clustering phenomena so that ${ }^{12} \mathrm{C}$ ion can be described as $\alpha$-cluster structures and the break-up of ${ }^{12} \mathrm{C}$ into three $\alpha$ particles is extremely probable. 

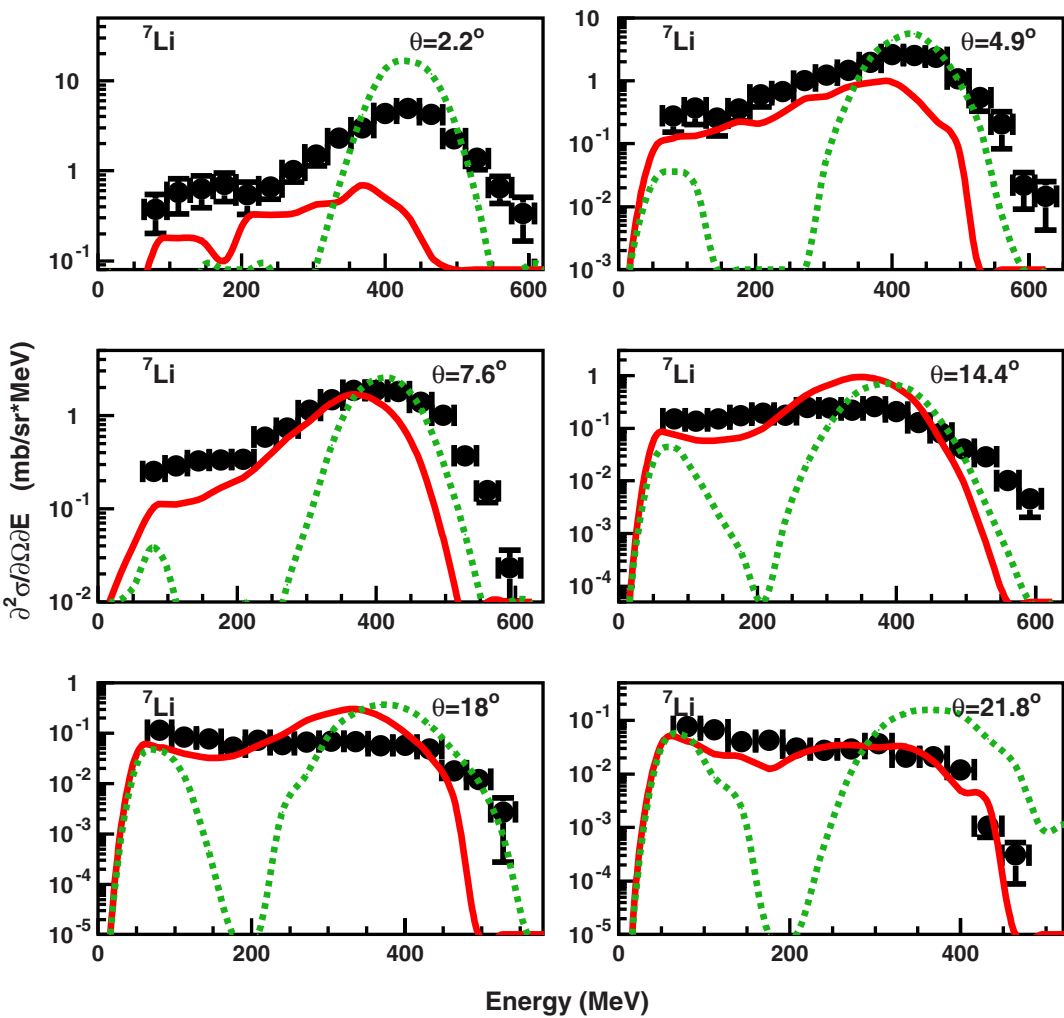

Figure 7. Double-differential cross sections measured at $\theta_{\text {lab }}=2.2^{\circ}, 4.9^{\circ}, 7.6^{\circ}, 14.4^{\circ}, 18^{\circ}$ and $21.8^{\circ}$ for ${ }^{7} \mathrm{Li}$ produced in the ${ }^{12} \mathrm{C}+{ }^{12} \mathrm{C}$ reaction at $62 \mathrm{~A} \mathrm{MeV}$. The data are compared with the Binary Light Ion Cascade (dashed lines) and the QMD (solid lines) predictions.

\section{GeanT4 simulations}

The measured cross sections have been used to evaluate the performance of the nuclear reaction models implemented in GEANT4 in the region of intermediate energies and in the optimum experimental condition of a thin target. GEANT4 is an object-oriented toolkit to simulate the passage of particles through matter. It is currently used in a variety of physics domains, including medical physics, since it provides interaction models for electromagnetic and nuclear processes involved in the transport of therapeutic ion beams.

Nucleus-nucleus collisions, pre-equilibrium and de-excitation stages can be described by different models in GEANT4. At present, two models are potentially able to provide a realistic description of light ions' inelastic interactions in the energy range of interest for this work: G4BinaryLightIonReaction and G4QMDReaction.

The G4BinaryLightIonReaction model is based on the Binary Cascade approach (BIC) (Folger et al 2004). Although the suggested energy range of the model is $80 \mathrm{~A} \mathrm{MeV}-10$ A GeV (Folger et al 2004), ${ }^{13}$ its reference physics lists implement the model down to zero energy.

G4QMDReaction is a new native GEANT4 implementation (Koi 2010) of the general Quantum Molecular Dynamic model (QMD) (Niita et al 1995, Aichelin 1991). The set of

\footnotetext{
${ }^{13}$ http://geant4.web.cern.ch/geant4/support/proc_mod_catalog/models/hadronic/G4BinaryLightIonReaction.html.
} 

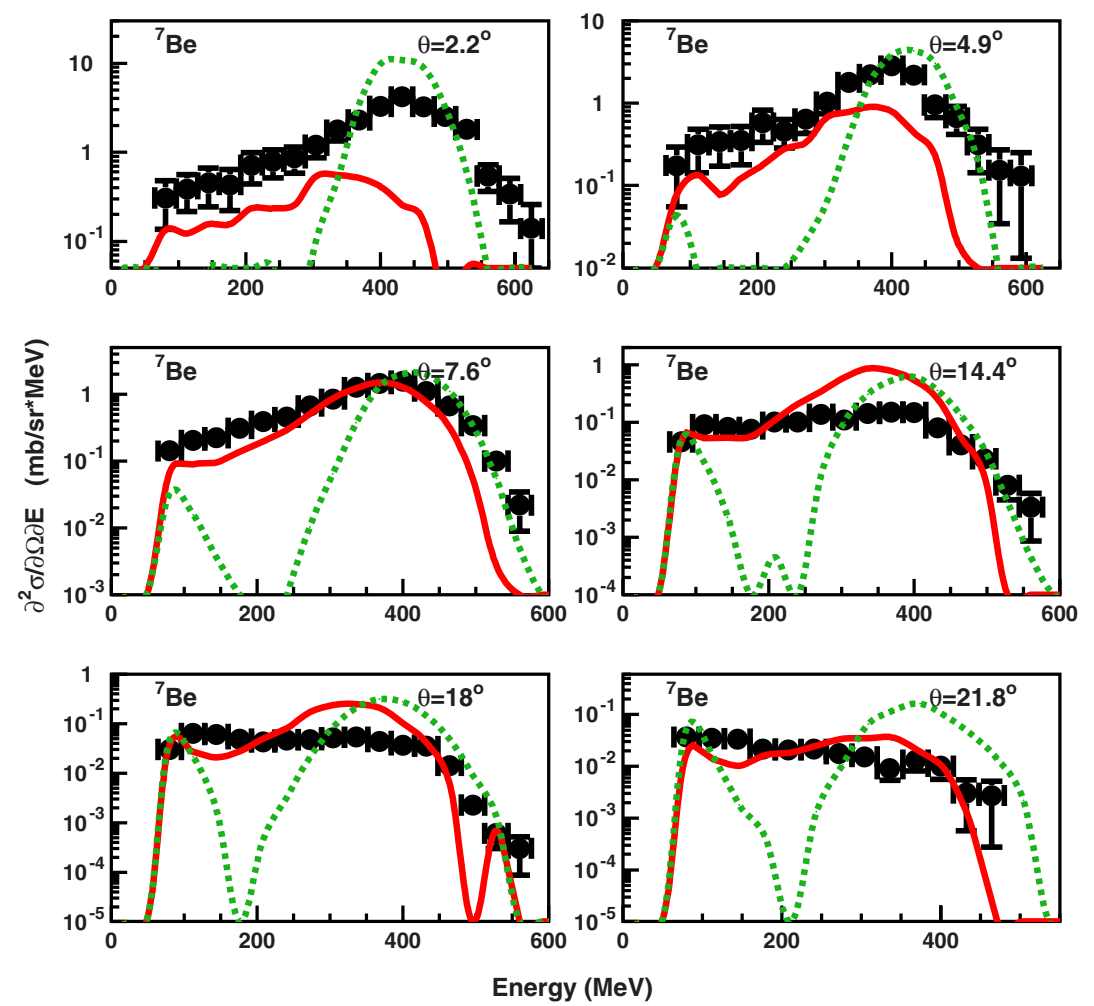

Figure 8. Double-differential cross sections measured at $\theta_{\mathrm{lab}}=2.2^{\circ}, 4.9^{\circ}, 7.6^{\circ}, 14.4^{\circ}, 18^{\circ}$ and $21.8^{\circ}$ for ${ }^{7} \mathrm{Be}$ produced in the ${ }^{12} \mathrm{C}+{ }^{12} \mathrm{C}$ reaction at $62 \mathrm{~A} \mathrm{MeV}$. The data are compared with the Binary Light Ion Cascade (dashed lines) and the QMD (solid lines) predictions.

parameters in the QMD model had been originally optimized to reproduce data in the energy range between $100 \mathrm{~A} \mathrm{MeV}$ and $3 \mathrm{~A} \mathrm{GeV}$. In order to test the QMD model in the energy range of interest for this work, the reference physics list had to be manually edited with respect to the version provided with GEANT4.

The simulations reported in this work have been performed with the version 9.4.p01 of GEANT4. In particular, in the simulation both the ${ }^{12} \mathrm{C}$ beam spot and the energy straggling in the target have been considered. In order to reduce the calculation time of the simulation, the inelastic interaction cross section of the ions was artificially increased by a factor of 100. The scaling factor has been chosen such that the thin-target approximation is still valid, namely the probability of having two or more inelastic interactions within the target remains negligible. A total statistic of a few $10^{12}$ events has been generated for each of the two physics models considered. Finally, the results of the simulations have been processed by an analytical algorithm to reproduce angular resolution, geometrical efficiency, energy resolution and energy thresholds of the detectors.

\section{Comparison of the experimental cross sections with GEANT 4 predictions}

Figures 5-11 show the comparison between the double-differential cross sections of the $Z>1$ fragments with the BIC (dashed lines) and the QMD (solid lines) model predictions at different emission angles. 

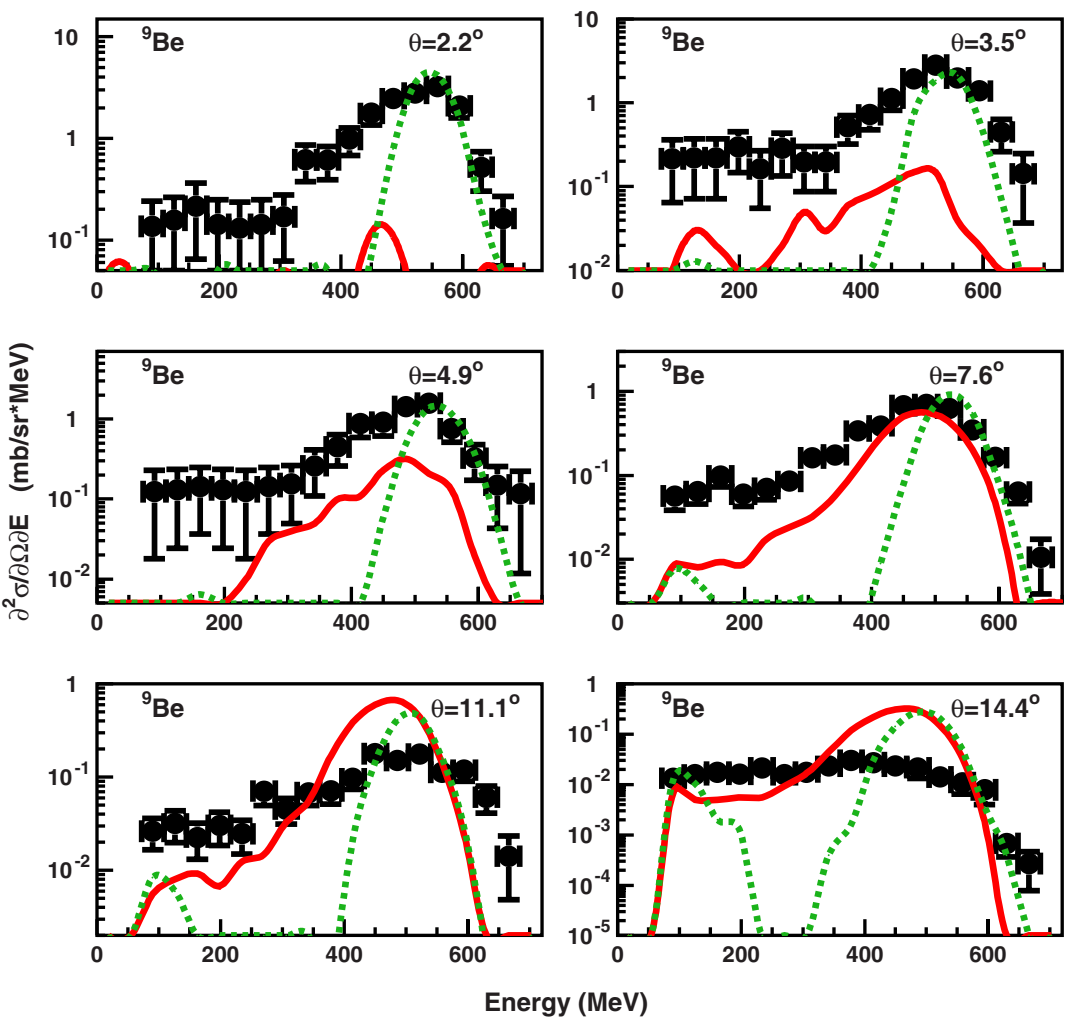

Figure 9. Double-differential cross sections measured at $\theta_{\text {lab }}=2.2^{\circ}, 3.5^{\circ}, 4.9^{\circ}, 7.6^{\circ}, 11.1^{\circ}$ and $14.4^{\circ}$ for ${ }^{9} \mathrm{Be}$ produced in the ${ }^{12} \mathrm{C}+{ }^{12} \mathrm{C}$ reaction at $62 \mathrm{~A} \mathrm{MeV}$. The data are compared with the Binary Light Ion Cascade (dashed lines) and the QMD (solid lines) predictions.

Since the experimental results have been compared with the code predictions for many different isotopes in a wide angular range, some general conclusions can be drawn for the two models. As one can see, e.g., from figure 5, the BIC model better reproduces the cross sections of the fragments emitted at high energies and small angles, i.e. the fragments produced in peripheral collisions, rather than those of the fragments emitted at low energies. A possible explanation could be that in the BIC model a time-invariant optical potential is used. Indeed, this approach seems to be more appropriate for treating peripheral reactions, where the dynamical evolution of the potential is slightly affected by the nucleon scatterings, with respect to the central ones. Moreover, the BIC model assumption that the participant nucleons, i.e. nucleons in the projectile and the ones scattered in the cascade, cannot collide with each other may have a minor effect on the description of peripheral reactions than the central ones. As has been pointed out by Folger et al (2004), the BIC applicability is limited to small participant densities. In contrast, the QMD model better reproduces the low-energy part of the spectra, i.e. the fragments produced in more dissipative central collisions. Indeed, in the QMD model, unlike the BIC, all nucleons, tracked simultaneously, can collide to each other and the potential dynamically changes along with the time evolution of the nucleons. Based on these assumptions, the QMD model seems to be more suitable to describe central reactions where the participant-participant collisions and the dynamical evolution of the potential become more important. 

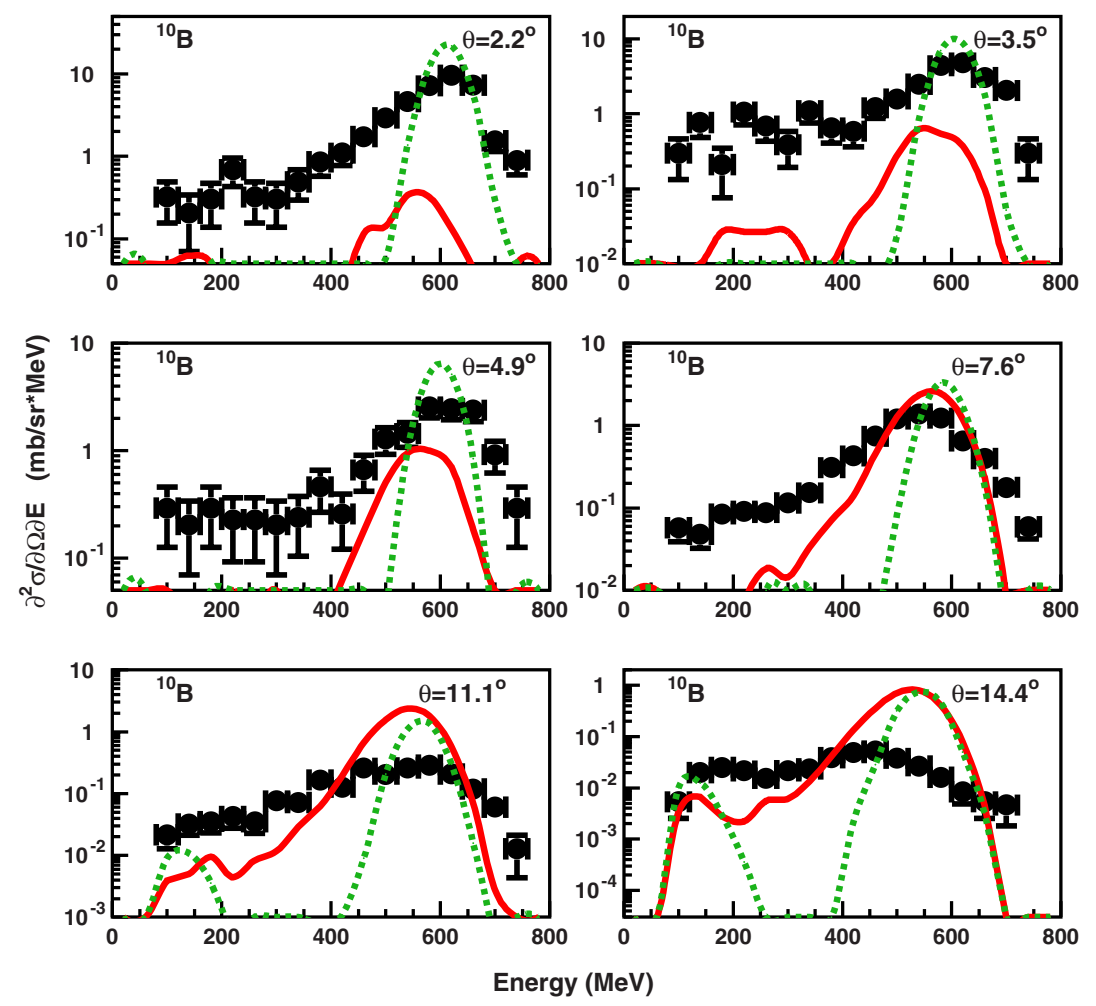

Figure 10. Double-differential cross sections measured at $\theta_{\text {lab }}=2.2^{\circ}, 3.5^{\circ}, 4.9^{\circ}, 7.6^{\circ}, 11.1^{\circ}$ and $14.4^{\circ}$ for ${ }^{10} \mathrm{~B}$ produced in the ${ }^{12} \mathrm{C}+{ }^{12} \mathrm{C}$ reaction at $62 \mathrm{~A} \mathrm{MeV}$. The data are compared with the Binary Light Ion Cascade (dashed lines) and the QMD (solid lines) predictions.

In figures $12-14$, the double-differential cross sections of the $Z=1$ isotopes are compared with the BIC (dashed lines) and the QMD (solid lines) predictions. As has been observed in the $Z>1$ cases, the high-energy region of the distributions at small angles is better reproduced by the BIC model rather than the QMD.

The comparison between the experimental angular distributions of all fragments and the two model predictions are shown in figure 15. As one can clearly see, the angular distributions of the fragment production cross sections are better approximated by the BIC calculations than by the QMD ones. Large discrepancies between the experimental cross sections and both model predictions can be observed. Indeed, differences up to a factor of 5 have been observed in the comparison with the BIC calculations, whereas discrepancies up to one order of magnitude have been found between the experimental data and the QMD.

In particular, for the $Z>1$ fragments, the experimental production cross sections are underestimated at most forward angles by the QMD and overestimated at larger angles by both models. As has been observed from the comparison between the double-differential cross sections and the calculations (see, e.g., figure 5), at most forward angles the contribution predicted by the QMD to the high-energy part of the energy distributions is smaller than the experimental cross section. On the other hand, at larger angles, both BIC and QMD predict a Gaussian-like peak that is not present in the experimental data (see, e.g., figure 9).

Böhlen et al (2010) have also observed that at higher energies (100-500 A MeV) the experimental cross sections are underestimated at most forward angles and overestimated at larger angles by both model predictions. However, contrary to what has been observed in 

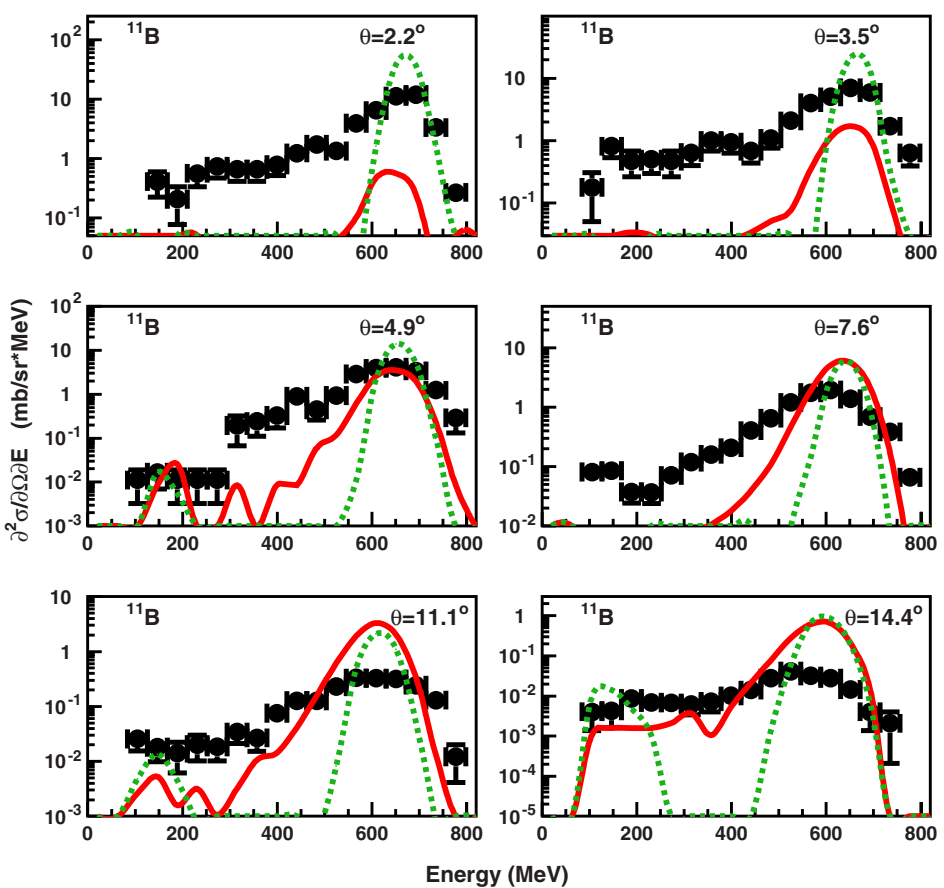

Figure 11. Double-differential cross sections measured at $\theta_{\text {lab }}=2.2^{\circ}, 3.5^{\circ}, 4.9^{\circ}, 7.6^{\circ}, 11.1^{\circ}$ and $14.4^{\circ}$ for ${ }^{11} \mathrm{~B}$ produced in the ${ }^{12} \mathrm{C}+{ }^{12} \mathrm{C}$ reaction at $62 \mathrm{~A} \mathrm{MeV}$. The data are compared with the Binary Light Ion Cascade (dashed lines) and the QMD (solid lines) predictions.
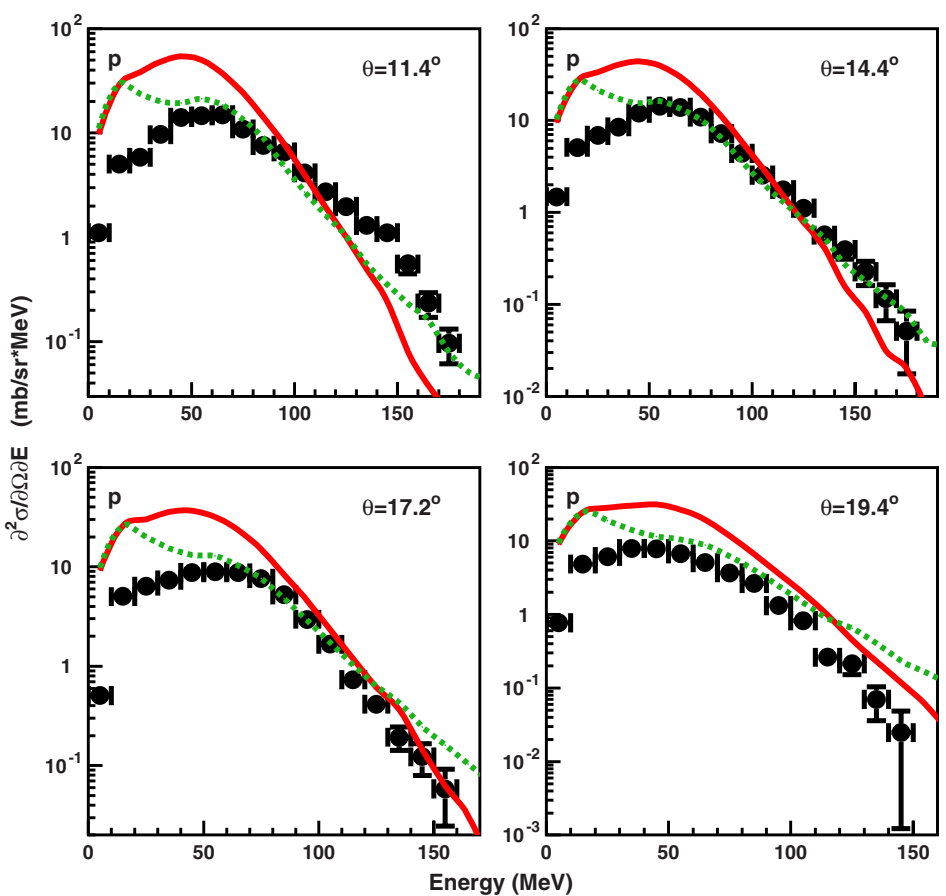

Figure 12. Double-differential cross sections measured at $\theta_{\text {lab }}=11.4^{\circ}, 14.4^{\circ}, 17.2^{\circ}$ and $19.4^{\circ}$ for protons produced in the ${ }^{12} \mathrm{C}+{ }^{12} \mathrm{C}$ reaction at $62 \mathrm{~A} \mathrm{MeV}$. The data are compared with the Binary Light Ion Cascade (dashed lines) and the QMD (solid lines) predictions. 

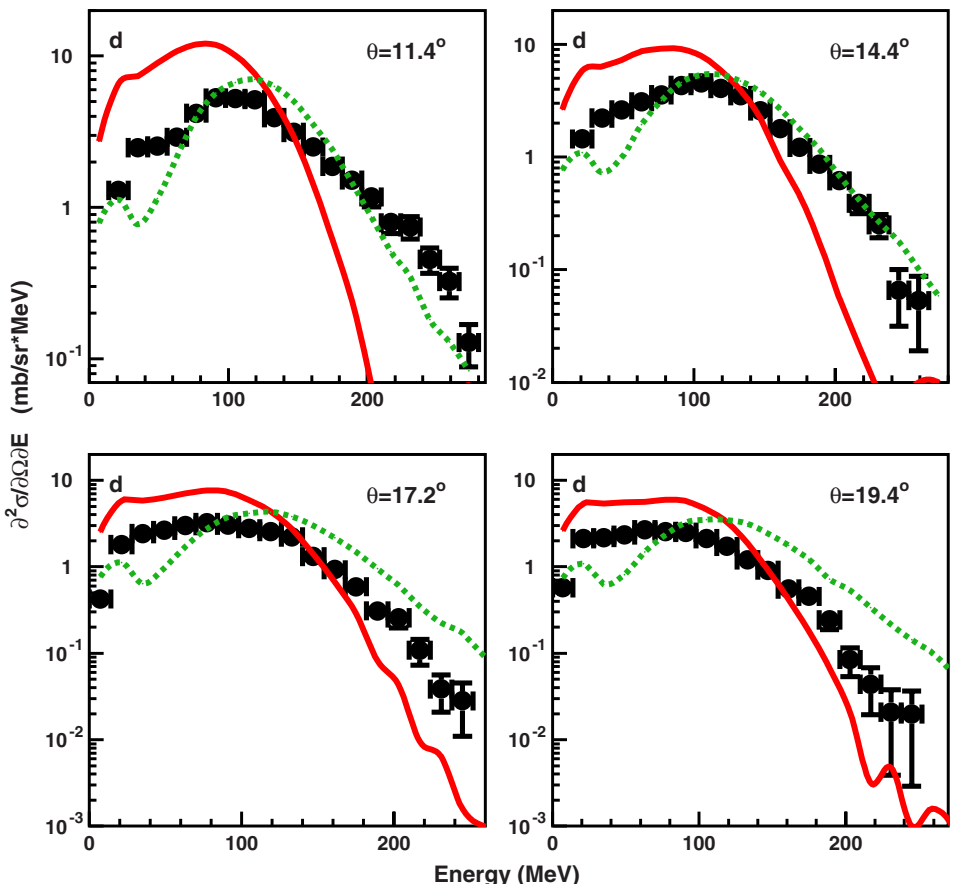

Figure 13. Double-differential cross sections measured at $\theta_{l a b}=11.4^{\circ}, 14.4^{\circ}, 17.2^{\circ}$ and $19.4^{\circ}$ for deuterons produced in the ${ }^{12} \mathrm{C}+{ }^{12} \mathrm{C}$ reaction at $62 \mathrm{~A} \mathrm{MeV}$. The data are compared with the Binary Light Ion Cascade (dashed lines) and the QMD (solid lines) predictions.
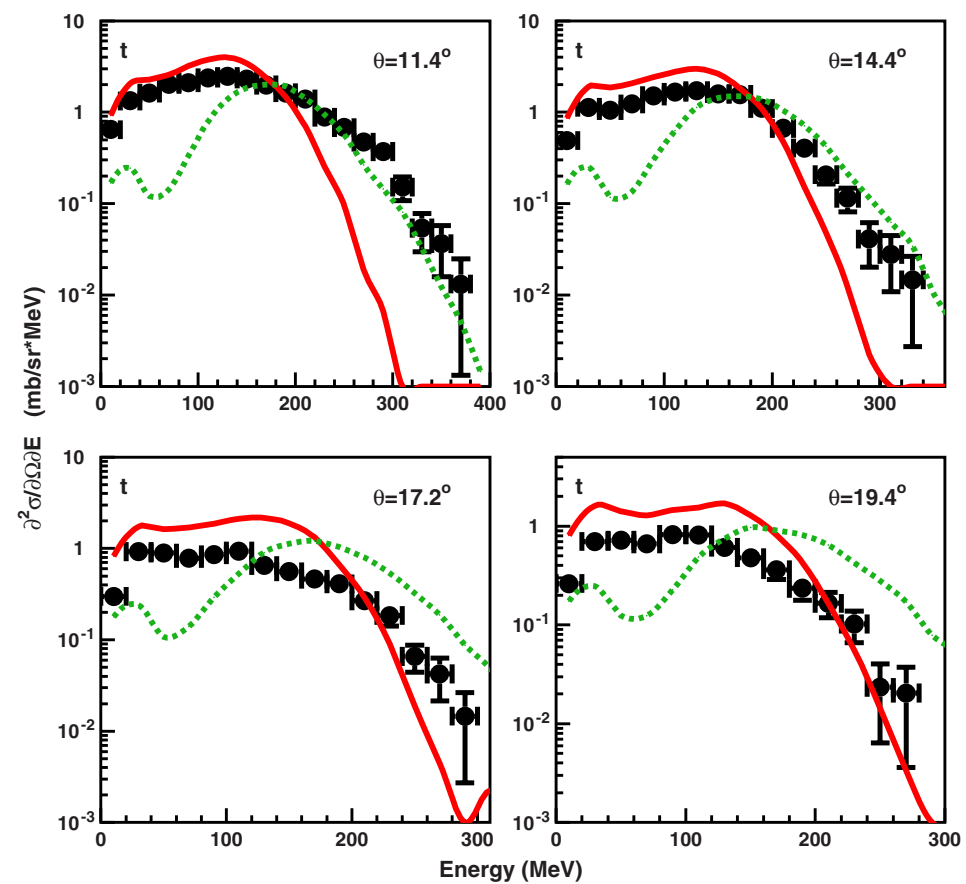

Figure 14. Double-differential cross sections measured at $\theta_{\text {lab }}=11.4^{\circ}, 14.4^{\circ}, 17.2^{\circ}$ and $19.4^{\circ}$ for tritons produced in the ${ }^{12} \mathrm{C}+{ }^{12} \mathrm{C}$ reaction at $62 \mathrm{~A} \mathrm{MeV}$. The data are compared with the Binary Light Ion Cascade (dashed lines) and the QMD (solid lines) predictions. 

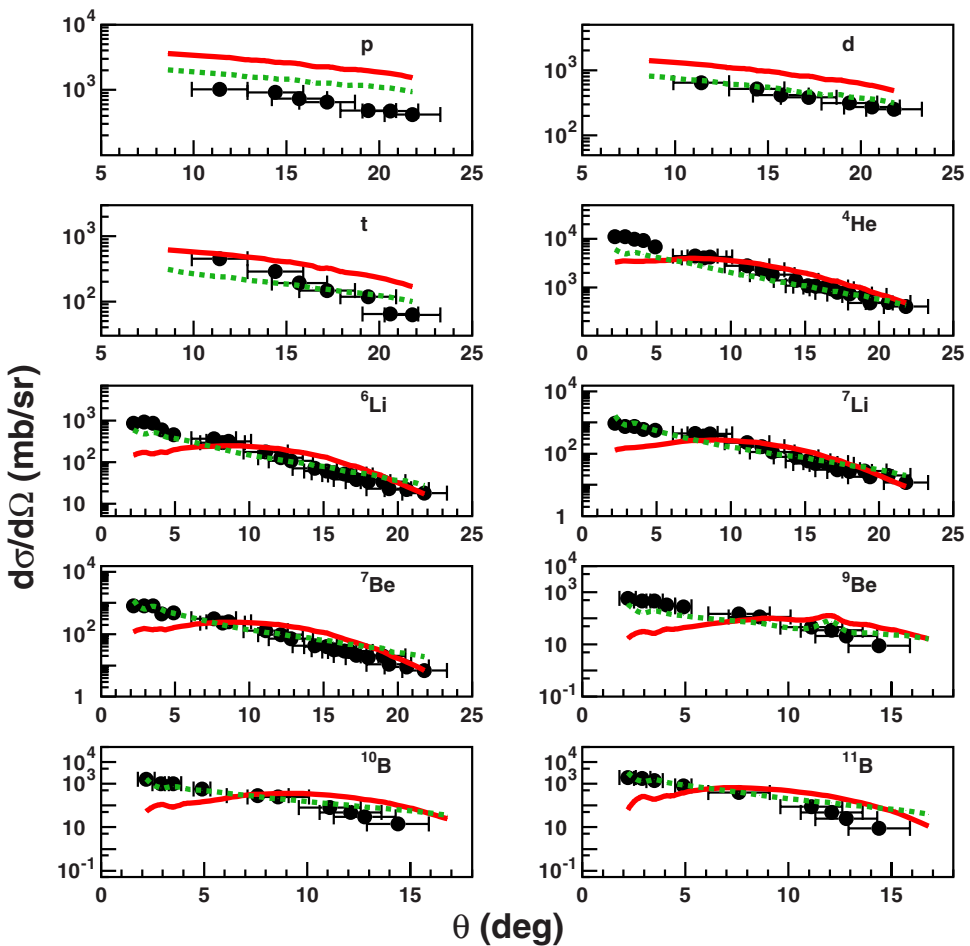

Figure 15. Angular distributions of all the detected fragments compared with the predictions of the Binary Light Ion Cascade (dashed lines) and QMD (solid lines).

our case, Böhlen et al (2010) conclude that the QMD model is more suitable to predict the fragment yields with respect to the BIC one. A possible reason is that in this work the QMD model has been applied in an energy region outside its reference energy range, where it was neither tested nor designed to work properly.

\section{Summary and conclusions}

This work presents extensive measurements of carbon fragmentation cross sections that are necessary to benchmark the Monte Carlo codes for their use in hadrontherapy. In the current analysis, the measured cross sections have been used to validate two GEANT4 nuclear reaction models: the G4BinaryLightIonReaction (BIC) and G4QMDReaction (QMD).

The results show that the QMD model approximates the low-energy region of the doubledifferential cross sections better than the BIC one. The latter, on the other hand, gives better results with respect to the QMD in reproducing the fragmentation peak, i.e. the largest contribution to the double-differential cross sections. As a consequence, the integrated doubledifferential cross sections as a function of the angle, i.e. the angular distributions, are better approximated by the BIC model than the QMD one. However, it has to be noted that the energy at which the present fragmentation measurements have been performed is lower than the energy range where the QMD model has been tested and designed to work.

In conclusion, even though the comparison between the measured cross sections and the GEANT4 predictions shows promising results, the prediction capability of both models needs to be improved at intermediate energies. Moreover, high-quality systematic measurements 
of double-differential cross sections with different projectile-target-energy combinations are required to improve the nuclear reaction models of the Monte Carlo codes for their use in hadrontherapy.

\section{Appendix}

Table A1. Double-differential cross sections of ${ }^{4} \mathrm{He}$ with respect to the emission angle (columns) and kinetic energy (rows). The values in parentheses represent the uncertainties in the last digits.

\begin{tabular}{lclllll}
\hline $\begin{array}{l}{ }^{4} \mathrm{He} \\
\text { Energy }\end{array}$ & $\begin{array}{l}\theta_{\mathrm{lab}}=2.2^{\circ} \\
\partial^{2} \sigma / \partial \Omega \partial E\end{array}$ & $\begin{array}{l}\theta_{\mathrm{lab}}=4.9^{\circ} \\
\partial^{2} \sigma / \partial \Omega \partial E\end{array}$ & $\begin{array}{l}\theta_{\mathrm{lab}}=7.6^{\circ} \\
\partial^{2} \sigma / \partial \Omega \partial E \\
\mathrm{mb})\end{array}$ & $\begin{array}{l}\theta_{\mathrm{lab}}=14.4^{\circ} \\
\partial^{2} \sigma / \partial \Omega \partial E\end{array}$ & $\begin{array}{l}\theta_{\mathrm{lab}}=18^{\circ} \\
\partial^{2} \sigma / \partial \Omega \partial E\end{array}$ & $\begin{array}{l}\theta_{\mathrm{lab}}=21.8^{\circ} \\
\partial^{2} \sigma / \partial \Omega \partial E \\
\mathrm{mb} /(\mathrm{sr} \mathrm{MeV})\end{array}$ \\
\hline $30(10)$ & $5.0(9)$ & $3.8(7)$ & $3.2(3)$ & $2.8(2)$ & $1.8(2)$ & $2.0(2)$ \\
$50(10)$ & $5.2(8)$ & $4.4(7)$ & $3.7(3)$ & $3.6(3)$ & $2.8(2)$ & $2.4(2)$ \\
$70(10)$ & $6.5(9)$ & $7(1)$ & $5.0(4)$ & $3.6(3)$ & $2.6(2)$ & $2.1(2)$ \\
$90(10)$ & $9(1)$ & $6.4(8)$ & $5.7(5)$ & $4.0(3)$ & $2.4(2)$ & $1.9(2)$ \\
$110(10)$ & $11(1)$ & $8(1)$ & $7.2(6)$ & $4.6(4)$ & $2.5(2)$ & $1.9(2)$ \\
$130(10)$ & $15(2)$ & $12(1)$ & $10.3(9)$ & $5.9(5)$ & $2.8(2)$ & $1.9(2)$ \\
$150(10)$ & $24(3)$ & $16(2)$ & $14(1)$ & $6.5(5)$ & $2.9(3)$ & $1.7(1)$ \\
$170(10)$ & $32(3)$ & $24(3)$ & $20(2)$ & $7.2(6)$ & $3.0(3)$ & $1.5(1)$ \\
$190(10)$ & $53(5)$ & $33(3)$ & $27(2)$ & $7.0(6)$ & $3.2(3)$ & $1.4(1)$ \\
$210(10)$ & $72(7)$ & $42(4)$ & $33(3)$ & $6.1(5)$ & $3.1(3)$ & $1.2(1)$ \\
$230(10)$ & $93(9)$ & $51(5)$ & $35(3)$ & $4.5(4)$ & $2.8(2)$ & $0.86(8)$ \\
$250(10)$ & $107(10)$ & $49(5)$ & $27(2)$ & $2.7(2)$ & $2.2(2)$ & $0.58(6)$ \\
$270(10)$ & $66(6)$ & $34(3)$ & $16(1)$ & $1.5(1)$ & $1.7(2)$ & $0.34(4)$ \\
$290(10)$ & $32(3)$ & $27(3)$ & $7.8(7)$ & $0.73(7)$ & $1.1(1)$ & $0.15(2)$ \\
$310(10)$ & $17(2)$ & $15(2)$ & $3.3(3)$ & $0.33(4)$ & $0.63(6)$ & $0.11(2)$ \\
$330(10)$ & $8(1)$ & $5.2(9)$ & $1.2(1)$ & $0.12(2)$ & $0.37(4)$ & $0.044(9)$ \\
$350(10)$ & $4.4(8)$ & $2.6(5)$ & $0.43(6)$ & $0.05(1)$ & $0.20(3)$ & $0.024(7)$ \\
$370(10)$ & $1.5(4)$ & $1.1(3)$ & $0.21(4)$ & $0.017(5)$ & $0.08(1)$ & $0.0027(8)$ \\
$390(10)$ & $0.8(3)$ & $0.5(3)$ & $0.11(3)$ & $0.0022(7)$ & $0.034(5)$ & $0.0015(6)$ \\
\hline
\end{tabular}

Table A2. Double-differential cross sections of ${ }^{6} \mathrm{Li}$ with respect to the emission angle (columns) and kinetic energy (rows). The values in parentheses represent the uncertainties in the last digits.

\begin{tabular}{lllllll}
\hline${ }^{6} \mathrm{Li}$ & $\theta_{\mathrm{lab}}=2.2^{\circ}$ & $\begin{array}{l}\theta_{\mathrm{lab}}=4.9^{\circ} \\
\partial^{2} \sigma / \partial \Omega \partial E\end{array}$ & $\begin{array}{l}\theta_{\mathrm{lab}}=7.6^{\circ} \\
\partial^{2} \sigma / \partial \Omega \partial E \\
\mathrm{mb} /(\mathrm{sr} \mathrm{MeV})\end{array}$ & $\begin{array}{l}\theta_{\mathrm{lab}}=14.4^{\circ} \sigma / \partial \Omega \partial E \\
\mathrm{mb} /(\mathrm{sr} \mathrm{MeV})\end{array}$ & $\begin{array}{l}\theta_{\mathrm{lab}}=18^{\circ} \\
\partial^{2} \sigma / \partial \Omega \partial E \\
\mathrm{mb} /(\mathrm{sr} \mathrm{MeV})\end{array}$ & $\begin{array}{l}\theta_{\mathrm{lab}}=21.8^{\circ} \\
\mathrm{mb} / \partial \Omega \partial E\end{array}$ \\
\hline $\mathrm{mb} /(\mathrm{sr} \mathrm{MeV})$ & $\mathrm{meV} /(\mathrm{sr} \mathrm{MeV})$ & $\mathrm{MeV})$ \\
$45(15)$ & $0.4(2)$ & $0.09(3)$ & $0.12(2)$ & $0.042(7)$ & $0.032(7)$ & $0.05(1)$ \\
$75(15)$ & $0.4(2)$ & $0.14(3)$ & $0.35(5)$ & $0.17(2)$ & $0.13(2)$ & $0.14(2)$ \\
$105(15)$ & $0.4(2)$ & $0.18(4)$ & $0.33(5)$ & $0.17(2)$ & $0.12(2)$ & $0.09(1)$ \\
$135(15)$ & $0.5(2)$ & $0.14(3)$ & $0.47(7)$ & $0.15(2)$ & $0.09(1)$ & $0.06(1)$ \\
$165(15)$ & $1.0(3)$ & $0.5(2)$ & $0.43(6)$ & $0.18(2)$ & $0.11(2)$ & $0.07(1)$ \\
$195(15)$ & $0.7(2)$ & $0.8(3)$ & $0.59(7)$ & $0.17(2)$ & $0.11(2)$ & $0.05(1)$ \\
$225(15)$ & $1.4(4)$ & $0.9(3)$ & $0.79(9)$ & $0.20(2)$ & $0.10(1)$ & $0.049(9)$ \\
$255(15)$ & $1.6(4)$ & $1.1(3)$ & $1.0(1)$ & $0.21(2)$ & $0.10(1)$ & $0.036(7)$ \\
$285(15)$ & $2.3(5)$ & $1.6(3)$ & $1.4(1)$ & $0.24(3)$ & $0.08(1)$ & $0.021(4)$ \\
$315(15)$ & $3.0(5)$ & $2.3(5)$ & $1.5(2)$ & $0.22(3)$ & $0.07(1)$ & $0.028(7)$ \\
$345(15)$ & $4.7(8)$ & $2.4(5)$ & $1.8(2)$ & $0.22(3)$ & $0.08(1)$ & $0.011(4)$ \\
$375(15)$ & $4.7(8)$ & $2.1(4)$ & $1.5(2)$ & $0.18(2)$ & $0.06(1)$ & $0.010(4)$ \\
$405(15)$ & $4.0(7)$ & $1.4(3)$ & $1.1(1)$ & $0.12(2)$ & $0.034(7)$ & $0.0020(6)$ \\
$435(15)$ & $2.5(6)$ & $0.9(3)$ & $0.58(7)$ & $0.047(9)$ & $0.029(8)$ & $0.0008(4)$ \\
$465(15)$ & $1.0(3)$ & $0.4(1)$ & $0.28(4)$ & $0.025(6)$ & $0.008(4)$ & $/$ \\
$495(15)$ & $0.4(2)$ & $0.2(1)$ & $0.10(2)$ & $0.009(4)$ & $0.004(3)$ & $/$ \\
$525(15)$ & $0.2(1)$ & $0.02(1)$ & $0.02(1)$ & $0.006(4)$ & $0.0031(3)$ & $/$ \\
$555(15)$ & $0.2(1)$ & $0.02(1)$ & $0.02(1)$ & $0.0031(26)$ & & $/$ \\
\hline
\end{tabular}


Table A3. Double-differential cross sections of ${ }^{7} \mathrm{Li}$ with respect to the emission angle (columns) and kinetic energy (rows). The values in parentheses represent the uncertainties in the last digits.

\begin{tabular}{|c|c|c|c|c|c|c|}
\hline $\begin{array}{l}{ }^{7} \mathrm{Li} \\
\text { Energy } \\
(\mathrm{MeV})\end{array}$ & $\begin{array}{l}\theta_{\text {lab }}=2.2^{\circ} \\
\partial^{2} \sigma / \partial \Omega \partial E \\
\mathrm{mb} /(\operatorname{sr} \mathrm{MeV})\end{array}$ & $\begin{array}{l}\theta_{\mathrm{lab}}=4.9^{\circ} \\
\partial^{2} \sigma / \partial \Omega \partial E \\
\mathrm{mb} /(\mathrm{sr} \mathrm{MeV})\end{array}$ & $\begin{array}{l}\theta_{\mathrm{lab}}=7.6^{\circ} \\
\partial^{2} \sigma / \partial \Omega \partial E \\
\mathrm{mb} /(\mathrm{sr} \mathrm{MeV})\end{array}$ & $\begin{array}{l}\theta_{\mathrm{lab}}=14.4^{\circ} \\
\partial^{2} \sigma / \partial \Omega \partial E \\
\mathrm{mb} /(\mathrm{sr} \mathrm{MeV})\end{array}$ & $\begin{array}{l}\theta_{\mathrm{lab}}=18^{\circ} \\
\partial^{2} \sigma / \partial \Omega \partial E \\
\mathrm{mb} /(\operatorname{sr} \mathrm{MeV})\end{array}$ & $\begin{array}{l}\theta_{\mathrm{lab}}=21.8^{\circ} \\
\partial^{2} \sigma / \partial \Omega \partial E \\
\mathrm{mb} /(\mathrm{sr} \mathrm{MeV})\end{array}$ \\
\hline 80 (16) & $0.4(2)$ & $0.3(1)$ & $0.25(4)$ & $0.15(2)$ & $0.11(2)$ & 0.08 (1) \\
\hline $112(16)$ & $0.6(2)$ & $0.4(2)$ & $0.29(5)$ & $0.14(2)$ & $0.08(1)$ & 0.07 (1) \\
\hline $144(16)$ & $0.6(2)$ & $0.3(1)$ & $0.33(6)$ & $0.15(2)$ & 0.08 (1) & $0.040(7)$ \\
\hline $176(16)$ & $0.7(2)$ & $0.4(1)$ & $0.34(5)$ & $0.18(2)$ & 0.05 (1) & $0.042(8)$ \\
\hline $208(16)$ & $0.5(2)$ & $0.6(2)$ & $0.34(5)$ & 0.19 (2) & 0.07 (1) & $0.030(7)$ \\
\hline $240(16)$ & $0.7(2)$ & $0.7(2)$ & $0.59(8)$ & $0.18(2)$ & $0.06(1)$ & $0.028(6)$ \\
\hline 272 (16) & $1.0(3)$ & $1.0(3)$ & $0.7(1)$ & $0.25(3)$ & 0.07 (1) & $0.030(7)$ \\
\hline 304 (16) & $1.5(4)$ & $1.2(3)$ & $1.1(1)$ & $0.24(3)$ & 0.07 (1) & 0.037 (9) \\
\hline $336(16)$ & $2.3(4)$ & $1.5(3)$ & $1.5(2)$ & $0.22(3)$ & 0.07 (1) & $0.021(5)$ \\
\hline 368 (16) & $3.0(5)$ & $2.0(4)$ & $1.9(2)$ & $0.26(3)$ & $0.06(1)$ & $0.021(6)$ \\
\hline $400(16)$ & $4.3(7)$ & $2.6(5)$ & $1.9(2)$ & $0.20(3)$ & $0.06(1)$ & $0.012(4)$ \\
\hline 432 (16) & $4.9(8)$ & $2.5(5)$ & $1.8(2)$ & $0.13(2)$ & $0.05(1)$ & $0.0011(4)$ \\
\hline 464 (16) & $4.2(7)$ & $2.4(5)$ & $1.4(2)$ & 0.08 (1) & $0.018(5)$ & $0.0003(2)$ \\
\hline 496 (16) & $2.3(4)$ & $1.1(3)$ & $1.0(1)$ & 0.04 (1) & $0.012(4)$ & I \\
\hline $528(16)$ & $1.4(3)$ & $0.5(2)$ & $0.37(7)$ & $0.03(1)$ & $0.003(2)$ & / \\
\hline $560(16)$ & $0.6(2)$ & $0.2(1)$ & $0.16(4)$ & $0.010(4)$ & I & l \\
\hline $592(16)$ & $0.3(2)$ & $0.02(1)$ & $0.02(1)$ & $0.005(3)$ & / & / \\
\hline
\end{tabular}

Table A4. Double-differential cross sections of ${ }^{7} \mathrm{Be}$ with respect to the emission angle (columns) and kinetic energy (rows). The values in parentheses represent the uncertainties in the last digits.

\begin{tabular}{|c|c|c|c|c|c|c|}
\hline $\begin{array}{l}{ }^{7} \mathrm{Be} \\
\text { Energy } \\
(\mathrm{MeV})\end{array}$ & $\begin{array}{l}\theta_{\text {lab }}=2.2^{\circ} \\
\partial^{2} \sigma / \partial \Omega \partial E \\
\mathrm{mb} /(\mathrm{sr} \mathrm{MeV})\end{array}$ & $\begin{array}{l}\theta_{\mathrm{lab}}=4.9^{\circ} \\
\partial^{2} \sigma / \partial \Omega \partial E \\
\mathrm{mb} /(\mathrm{sr} \mathrm{MeV})\end{array}$ & $\begin{array}{l}\theta_{\mathrm{lab}}=7.6^{\circ} \\
\partial^{2} \sigma / \partial \Omega \partial E \\
\mathrm{mb} /(\mathrm{sr} \mathrm{MeV})\end{array}$ & $\begin{array}{l}\theta_{\text {lab }}=14.4^{\circ} \\
\partial^{2} \sigma / \partial \Omega \partial E \\
\mathrm{mb} /(\mathrm{sr} \mathrm{MeV})\end{array}$ & $\begin{array}{l}\theta_{\mathrm{lab}}=18^{\circ} \\
\partial^{2} \sigma / \partial \Omega \partial E \\
\mathrm{mb} /(\mathrm{sr} \mathrm{MeV})\end{array}$ & $\begin{array}{l}\theta_{\text {lab }}=21.8^{\circ} \\
\partial^{2} \sigma / \partial \Omega \partial E \\
\mathrm{mb} /(\mathrm{sr} \mathrm{MeV})\end{array}$ \\
\hline $80(16)$ & $0.3(2)$ & $0.2(1)$ & $0.14(3)$ & $0.046(8)$ & $0.030(6)$ & $0.037(8)$ \\
\hline $112(16)$ & $0.4(2)$ & $0.3(2)$ & $0.20(4)$ & $0.09(1)$ & $0.06(1)$ & $0.035(6)$ \\
\hline 144 (16) & $0.5(2)$ & $0.3(2)$ & $0.22(4)$ & 0.08 (1) & $0.06(1)$ & $0.034(7)$ \\
\hline $176(16)$ & $0.4(2)$ & $0.4(2)$ & $0.31(5)$ & $0.08(1)$ & $0.049(9)$ & $0.022(5)$ \\
\hline 208 (16) & $0.7(3)$ & $0.6(2)$ & $0.39(6)$ & $0.10(2)$ & $0.043(8)$ & $0.021(5)$ \\
\hline $240(16)$ & $0.8(3)$ & $0.5(2)$ & $0.45(6)$ & $0.10(2)$ & 0.047 (9) & $0.022(6)$ \\
\hline 272 (16) & 0.9 (3) & $0.6(2)$ & $0.67(8)$ & $0.14(2)$ & $0.048(9)$ & 0.017 (5) \\
\hline 304 (16) & $1.2(3)$ & $1.0(3)$ & $0.8(1)$ & $0.11(2)$ & $0.05(1)$ & $0.015(5)$ \\
\hline $336(16)$ & $1.8(4)$ & $1.8(4)$ & $1.3(1)$ & $0.14(2)$ & $0.05(1)$ & 0.009 (4) \\
\hline 368 (16) & $2.3(5)$ & $2.2(5)$ & $1.5(2)$ & $0.15(2)$ & $0.044(9)$ & $0.013(5)$ \\
\hline $400(16)$ & $3.3(6)$ & $2.8(6)$ & $1.6(2)$ & $0.15(2)$ & $0.037(8)$ & $0.010(4)$ \\
\hline 432 (16) & $4.2(7)$ & $2.2(5)$ & $1.1(1)$ & 0.08 (1) & $0.035(8)$ & $0.003(2)$ \\
\hline 464 (16) & $3.2(6)$ & 0.9 (3) & $0.66(9)$ & $0.041(8)$ & $0.014(5)$ & 0.003 (2) \\
\hline 496 (16) & $2.5(5)$ & 0.7 (2) & $0.34(5)$ & $0.023(6)$ & 0.0023 (6) & I \\
\hline $528(16)$ & $1.8(4)$ & $0.3(2)$ & $0.10(3)$ & 0.008 (4) & $0.0006(3)$ & I \\
\hline $560(16)$ & $0.5(2)$ & $0.2(1)$ & 0.02 (1) & 0.003 (2) & $0.0003(2)$ & / \\
\hline $592(16)$ & $0.3(2)$ & $0.13(12)$ & I & I & I & / \\
\hline $624(16)$ & $0.14(12)$ & I & I & I & I & I \\
\hline
\end{tabular}


Table A5. Double-differential cross sections of ${ }^{9} \mathrm{Be}$ with respect to the emission angle (columns) and kinetic energy (rows). The values in parentheses represent the uncertainties in the last digits.

\begin{tabular}{|c|c|c|c|c|c|c|}
\hline $\begin{array}{l}{ }^{9} \mathrm{Be} \\
\text { Energy } \\
(\mathrm{MeV})\end{array}$ & $\begin{array}{l}\theta_{\text {lab }}=2.2^{\circ} \\
\partial^{2} \sigma / \partial \Omega \partial E \\
\mathrm{mb} /(\mathrm{sr} \mathrm{MeV})\end{array}$ & $\begin{array}{l}\theta_{\mathrm{lab}}=3.5^{\circ} \\
\partial^{2} \sigma / \partial \Omega \partial E \\
\mathrm{mb} /(\mathrm{sr} \mathrm{MeV})\end{array}$ & $\begin{array}{l}\theta_{\mathrm{lab}}=4.9^{\circ} \\
\partial^{2} \sigma / \partial \Omega \partial E \\
\mathrm{mb} /(\mathrm{sr} \mathrm{MeV})\end{array}$ & $\begin{array}{l}\theta_{\mathrm{lab}}=7.6^{\circ} \\
\partial^{2} \sigma / \partial \Omega \partial E \\
\mathrm{mb} /(\mathrm{sr} \mathrm{MeV})\end{array}$ & $\begin{array}{l}\theta_{\mathrm{lab}}=11.1^{\circ} \\
\partial^{2} \sigma / \partial \Omega \partial E \\
\mathrm{mb} /(\mathrm{sr} \mathrm{MeV})\end{array}$ & $\begin{array}{l}\theta_{\mathrm{lab}}=14.4^{\circ} \\
\partial^{2} \sigma / \partial \Omega \partial E \\
\mathrm{mb} /(\mathrm{sr} \mathrm{MeV})\end{array}$ \\
\hline $90(18)$ & $0.14(11)$ & $0.2(1)$ & $0.12(11)$ & $0.06(2)$ & $0.03(1)$ & $0.013(5)$ \\
\hline $126(18)$ & $0.2(1)$ & $0.2(1)$ & $0.13(11)$ & $0.06(2)$ & 0.03 (1) & $0.016(4)$ \\
\hline $162(18)$ & $0.2(1)$ & $0.2(1)$ & 0.14 (11) & $0.10(3)$ & 0.02 (1) & $0.018(5)$ \\
\hline $198(18)$ & $0.14(11)$ & $0.3(2)$ & $0.13(11)$ & $0.06(2)$ & $0.03(1)$ & $0.016(5)$ \\
\hline 234 (18) & $0.13(11)$ & $0.2(1)$ & $0.12(11)$ & 0.07 (2) & $0.02(1)$ & $0.021(6)$ \\
\hline 270 (18) & $0.14(11)$ & $0.3(2)$ & 0.14 (11) & $0.08(2)$ & 0.07 (2) & $0.016(5)$ \\
\hline 306 (18) & $0.2(1)$ & $0.2(1)$ & $0.2(1)$ & $0.16(3)$ & $0.05(1)$ & $0.018(5)$ \\
\hline 342 (18) & $0.6(2)$ & $0.2(1)$ & $0.3(2)$ & $0.18(3)$ & 0.07 (2) & $0.023(7)$ \\
\hline 378 (18) & $0.6(2)$ & $0.5(2)$ & $0.4(2)$ & $0.34(5)$ & 0.07 (2) & $0.030(8)$ \\
\hline 414 (18) & $1.0(3)$ & $0.7(2)$ & $0.9(3)$ & $0.39(6)$ & $0.10(2)$ & $0.027(7)$ \\
\hline 450 (18) & $1.8(4)$ & $1.1(3)$ & $0.9(3)$ & $0.68(9)$ & $0.18(4)$ & $0.023(7)$ \\
\hline 486 (18) & $2.5(5)$ & $1.9(4)$ & $1.4(4)$ & $0.70(9)$ & $0.15(3)$ & $0.022(8)$ \\
\hline $522(18)$ & $2.8(6)$ & $2.8(6)$ & $1.6(4)$ & $0.63(9)$ & 0.18 (4) & $0.014(5)$ \\
\hline $558(18)$ & $3.2(7)$ & $2.0(4)$ & $0.8(2)$ & $0.35(5)$ & $0.11(3)$ & $0.011(5)$ \\
\hline 594 (18) & $2.1(5)$ & $1.4(3)$ & $0.3(2)$ & $0.16(3)$ & $0.12(3)$ & $0.008(4)$ \\
\hline $630(18)$ & $0.5(2)$ & $0.4(2)$ & 0.15 (11) & $0.06(2)$ & $0.06(2)$ & 0.0007 (3) \\
\hline $666(18)$ & $0.2(1)$ & 0.1 (1) & $0.12(11)$ & $0.011(7)$ & $0.014(9)$ & $0.0003(2)$ \\
\hline
\end{tabular}

Table A6. Double-differential cross sections of ${ }^{10} \mathrm{~B}$ with respect to the emission angle (columns) and kinetic energy (rows). The values in parentheses represent the uncertainties in the last digits.

\begin{tabular}{|c|c|c|c|c|c|c|}
\hline $\begin{array}{l}{ }^{10} \mathrm{~B} \\
\text { Energy } \\
(\mathrm{MeV})\end{array}$ & $\begin{array}{l}\theta_{\text {lab }}=2.2^{\circ} \\
\partial^{2} \sigma / \partial \Omega \partial E \\
\mathrm{mb} /(\mathrm{sr} \mathrm{MeV})\end{array}$ & $\begin{array}{l}\theta_{\mathrm{lab}}=3.5^{\circ} \\
\partial^{2} \sigma / \partial \Omega \partial E \\
\mathrm{mb} /(\mathrm{sr} \mathrm{MeV})\end{array}$ & $\begin{array}{l}\theta_{\mathrm{lab}}=4.9^{\circ} \\
\partial^{2} \sigma / \partial \Omega \partial E \\
\mathrm{mb} /(\mathrm{sr} \mathrm{MeV})\end{array}$ & $\begin{array}{l}\theta_{\mathrm{lab}}=7.6^{\circ} \\
\partial^{2} \sigma / \partial \Omega \partial E \\
\mathrm{mb} /(\mathrm{sr} \mathrm{MeV})\end{array}$ & $\begin{array}{l}\theta_{\text {lab }}=11.1^{\circ} \\
\partial^{2} \sigma / \partial \Omega \partial E \\
\mathrm{mb} /(\mathrm{sr} \mathrm{MeV})\end{array}$ & $\begin{array}{l}\theta_{\text {lab }}=14.4^{\circ} \\
\partial^{2} \sigma / \partial \Omega \partial E \\
\mathrm{mb} /(\mathrm{sr} \mathrm{MeV})\end{array}$ \\
\hline $100(20)$ & $0.3(2)$ & $0.3(2)$ & $0.3(2)$ & $0.06(2)$ & 0.02 (1) & $0.005(3)$ \\
\hline $140(20)$ & 0.2 (1) & $0.8(3)$ & 0.2 (1) & 0.05 (2) & 0.03 (1) & $0.020(6)$ \\
\hline $180(20)$ & $0.3(2)$ & $0.2(1)$ & $0.3(2)$ & 0.08 (2) & 0.04 (1) & $0.025(7)$ \\
\hline $220(20)$ & 0.7 (3) & $1.1(3)$ & 0.2 (1) & 0.09 (2) & 0.04 (2) & $0.021(6)$ \\
\hline $260(20)$ & $0.3(2)$ & 0.7 (3) & 0.2 (1) & 0.09 (2) & 0.04 (1) & $0.015(5)$ \\
\hline $300(20)$ & $0.3(2)$ & $0.4(2)$ & 0.2 (1) & $0.12(2)$ & 0.08 (2) & $0.022(6)$ \\
\hline $340(20)$ & $0.5(2)$ & $1.1(3)$ & 0.2 (1) & $0.15(3)$ & 0.07 (2) & $0.023(6)$ \\
\hline $380(20)$ & 0.9 (3) & $0.6(2)$ & $0.5(2)$ & $0.31(5)$ & 0.17 (3) & 0.039 (9) \\
\hline $420(20)$ & $1.1(3)$ & $0.6(2)$ & 0.3 (1) & $0.43(6)$ & 0.13 (3) & 0.05 (1) \\
\hline $460(20)$ & $1.7(4)$ & $1.2(3)$ & 0.7 (2) & 0.7 (1) & $0.26(5)$ & $0.05(1)$ \\
\hline $500(20)$ & $2.9(6)$ & $1.6(4)$ & $1.3(4)$ & $1.2(2)$ & 0.21 (4) & $0.038(9)$ \\
\hline $540(20)$ & $4.6(8)$ & $2.5(5)$ & $1.4(4)$ & $1.4(2)$ & $0.26(5)$ & $0.027(7)$ \\
\hline $580(20)$ & $7(1)$ & $4.4(8)$ & $2.6(5)$ & $1.2(2)$ & $0.29(5)$ & $0.016(5)$ \\
\hline $620(20)$ & $10(1)$ & $4.8(8)$ & $1.6(4)$ & $0.65(9)$ & 0.21 (4) & 0.008 (4) \\
\hline $660(20)$ & 7 (1) & $3.1(6)$ & $2.4(5)$ & $0.40(6)$ & 0.12 (3) & 0.005 (3) \\
\hline $700(20)$ & $1.5(4)$ & $2.1(5)$ & $0.9(5)$ & $0.18(3)$ & $0.06(2)$ & $0.005(3)$ \\
\hline $740(20)$ & 0.9 (1) & $0.3(2)$ & $0.3(2)$ & $0.06(2)$ & $0.013(8)$ & I \\
\hline
\end{tabular}


Table A7. Double-differential cross sections of ${ }^{11} \mathrm{~B}$ with respect to emission angle (columns) and kinetic energy (rows). The values in parentheses represent the uncertainties in the last digits.

\begin{tabular}{|c|c|c|c|c|c|c|}
\hline $\begin{array}{l}{ }^{11} \mathrm{~B} \\
\text { Energy } \\
(\mathrm{MeV})\end{array}$ & $\begin{array}{l}\theta_{\mathrm{lab}}=2.2^{\circ} \\
\partial^{2} \sigma / \partial \Omega \partial E \\
\mathrm{mb} /(\mathrm{sr} \mathrm{MeV})\end{array}$ & $\begin{array}{l}\theta_{\mathrm{lab}}=3.5^{\circ} \\
\partial^{2} \sigma / \partial \Omega \partial E \\
\mathrm{mb} /(\mathrm{sr} \mathrm{MeV})\end{array}$ & $\begin{array}{l}\theta_{\mathrm{lab}}=4.9^{\circ} \\
\partial^{2} \sigma / \partial \Omega \partial E \\
\mathrm{mb} /(\mathrm{sr} \mathrm{MeV})\end{array}$ & $\begin{array}{l}\theta_{\mathrm{lab}}=7.6^{\circ} \\
\partial^{2} \sigma / \partial \Omega \partial E \\
\mathrm{mb} /(\mathrm{sr} \mathrm{MeV})\end{array}$ & $\begin{array}{l}\theta_{\mathrm{lab}}=11.1^{\circ} \\
\partial^{2} \sigma / \partial \Omega \partial E \\
\mathrm{mb} /(\mathrm{sr} \mathrm{MeV})\end{array}$ & $\begin{array}{l}\theta_{\mathrm{lab}}=14.4^{\circ} \\
\partial^{2} \sigma / \partial \Omega \partial E \\
\mathrm{mb} /(\mathrm{sr} \mathrm{MeV})\end{array}$ \\
\hline $105(21)$ & I & $0.2(1)$ & $0.011(8)$ & $0.08(2)$ & 0.03 (1) & $0.004(3)$ \\
\hline 147 (21) & $0.4(2)$ & 0.8 (3) & 0.02 (1) & 0.09 (2) & $0.018(8)$ & $0.004(3)$ \\
\hline $189(21)$ & 0.2 (1) & $0.5(2)$ & $0.011(8)$ & 0.04 (1) & $0.014(8)$ & 0.009 (3) \\
\hline $231(21)$ & $0.6(2)$ & $0.5(2)$ & $0.011(8)$ & 0.04 (1) & $0.02(1)$ & $0.007(3)$ \\
\hline $273(21)$ & 0.7 (3) & $0.5(2)$ & $0.011(8)$ & 0.07 (2) & 0.02 (1) & 0.007 (3) \\
\hline $315(21)$ & 0.7 (2) & $0.6(2)$ & $0.2(1)$ & $0.12(3)$ & 0.03 (1) & $0.006(3)$ \\
\hline 357 (21) & 0.7 (2) & $1.0(3)$ & 0.2 (1) & $0.16(3)$ & 0.03 (1) & $0.007(3)$ \\
\hline 399 (21) & $0.8(3)$ & 0.9 (3) & $0.3(2)$ & $0.21(4)$ & $0.08(2)$ & $0.010(4)$ \\
\hline $441(21)$ & $1.2(4)$ & 0.7 (2) & 0.9 (3) & $0.41(6)$ & $0.13(3)$ & $0.014(5)$ \\
\hline $483(21)$ & $1.7(4)$ & $1.1(3)$ & $0.4(2)$ & $0.65(9)$ & $0.13(3)$ & $0.028(7)$ \\
\hline $525(21)$ & $1.3(4)$ & $2.1(5)$ & 0.9 (3) & $1.2(2)$ & $0.23(4)$ & 0.04 (1) \\
\hline 567 (21) & $3.9(7)$ & $4.1(7)$ & $2.9(6)$ & $1.8(2)$ & $0.33(5)$ & $0.032(8)$ \\
\hline 609 (21) & $6(1)$ & $5.1(9)$ & $3.9(7)$ & $1.9(2)$ & $0.33(5)$ & $0.028(7)$ \\
\hline $651(21)$ & $11(2)$ & $7(1)$ & $4.1(8)$ & $1.4(2)$ & $0.32(5)$ & $0.014(5)$ \\
\hline 693 (21) & $12(2)$ & $6(1)$ & $3.4(7)$ & $0.70(9)$ & $0.24(5)$ & $0.004(3)$ \\
\hline 735 (21) & $3.3(6)$ & $1.7(4)$ & $1.2(4)$ & $0.39(6)$ & $0.13(3)$ & $0.004(3)$ \\
\hline 777 (21) & $0.27(4)$ & $0.6(2)$ & $0.3(2)$ & 0.07 (2) & $0.012(8)$ & / \\
\hline
\end{tabular}

Table A8. Double-differential cross sections of protons with respect to the emission angle (columns) and kinetic energy (rows). The values in parentheses represent the uncertainties in the last digits.

\begin{tabular}{|c|c|c|c|c|}
\hline $\begin{array}{l}\mathrm{p} \\
\text { Energy } \\
(\mathrm{MeV})\end{array}$ & $\begin{array}{l}\theta_{\text {lab }}=11.4^{\circ} \\
\partial^{2} \sigma / \partial \Omega \partial E \\
\mathrm{mb} /(\mathrm{sr} \mathrm{MeV})\end{array}$ & $\begin{array}{l}\theta_{\text {lab }}=14.4^{\circ} \\
\partial^{2} \sigma / \partial \Omega \partial E \\
\mathrm{mb} /(\mathrm{sr} \mathrm{MeV})\end{array}$ & $\begin{array}{l}\theta_{\mathrm{lab}}=17.2^{\circ} \\
\partial^{2} \sigma / \partial \Omega \partial E \\
\mathrm{mb} /(\mathrm{sr} \mathrm{MeV})\end{array}$ & $\begin{array}{l}\theta_{\mathrm{lab}}=19.4^{\circ} \\
\partial^{2} \sigma / \partial \Omega \partial E \\
\mathrm{mb} /(\mathrm{sr} \mathrm{MeV})\end{array}$ \\
\hline $5(5)$ & $1.1(1)$ & $1.5(2)$ & $0.51(8)$ & $0.8(1)$ \\
\hline $15(5)$ & $5.0(5)$ & $5.1(5)$ & $5.0(5)$ & $4.9(5)$ \\
\hline $25(5)$ & $5.9(5)$ & $6.9(6)$ & $6.4(6)$ & $6.1(6)$ \\
\hline $35(5)$ & $9.6(9)$ & $8.5(7)$ & $7.4(7)$ & $7.9(7)$ \\
\hline $45(5)$ & $14(1)$ & $12(1)$ & $8.7(8)$ & $7.9(8)$ \\
\hline $55(5)$ & $15(1)$ & $14(1)$ & $8.9(8)$ & $6.7(7)$ \\
\hline $65(5)$ & $15(1)$ & $14(1)$ & $8.7(8)$ & $5.1(6)$ \\
\hline $75(5)$ & $11(1)$ & $11(1)$ & $7.7(7)$ & $3.7(4)$ \\
\hline $85(5)$ & $7.6(8)$ & $7.2(7)$ & $5.2(5)$ & $2.6(3)$ \\
\hline $95(5)$ & $6.6(7)$ & $4.4(5)$ & $3.0(3)$ & $1.3(2)$ \\
\hline $105(5)$ & $4.1(5)$ & $2.5(3)$ & $1.7(2)$ & $0.8(1)$ \\
\hline $115(5)$ & $2.7(3)$ & $1.8(2)$ & 0.7 (1) & $0.26(6)$ \\
\hline $125(5)$ & $2.0(3)$ & $1.1(2)$ & $0.41(8)$ & $0.21(6)$ \\
\hline $135(5)$ & $1.3(2)$ & $0.6(1)$ & $0.19(5)$ & 0.07 (3) \\
\hline $145(5)$ & $1.1(2)$ & $0.40(9)$ & $0.12(4)$ & $0.025(24)$ \\
\hline $155(5)$ & $0.6(1)$ & $0.23(7)$ & $0.06(3)$ & / \\
\hline $165(5)$ & $0.23(6)$ & $0.12(5)$ & I & I \\
\hline $175(5)$ & $0.10(4)$ & $0.05(3)$ & / & l \\
\hline
\end{tabular}


Table A9. Double-differential cross sections of deuterons with respect to the emission angle (columns) and kinetic energy (rows). The values in parentheses represent the uncertainties in the last digits.

\begin{tabular}{|c|c|c|c|c|}
\hline $\begin{array}{l}\text { d } \\
\text { Energy } \\
(\mathrm{MeV})\end{array}$ & $\begin{array}{l}\theta_{\text {lab }}=11.4^{\circ} \\
\partial^{2} \sigma / \partial \Omega \partial E \\
\mathrm{mb} /(\mathrm{sr} \mathrm{MeV})\end{array}$ & $\begin{array}{l}\theta_{\text {lab }}=14.4^{\circ} \\
\partial^{2} \sigma / \partial \Omega \partial E \\
\mathrm{mb} /(\mathrm{sr} \mathrm{MeV})\end{array}$ & $\begin{array}{l}\theta_{\text {lab }}=17.2^{\circ} \\
\partial^{2} \sigma / \partial \Omega \partial E \\
\mathrm{mb} /(\mathrm{sr} \mathrm{MeV})\end{array}$ & $\begin{array}{l}\theta_{\mathrm{lab}}=19.4^{\circ} \\
\partial^{2} \sigma / \partial \Omega \partial E \\
\mathrm{mb} /(\operatorname{sr~MeV})\end{array}$ \\
\hline $7(7)$ & / & / & $0.42(6)$ & $0.57(8)$ \\
\hline $21(7)$ & $1.3(1)$ & $1.4(2)$ & $1.8(2)$ & $2.1(2)$ \\
\hline $35(7)$ & $2.5(2)$ & $2.2(2)$ & $2.4(3)$ & $2.2(2)$ \\
\hline $49(7)$ & $2.5(2)$ & $2.6(2)$ & $2.6(3)$ & $2.3(2)$ \\
\hline $63(7)$ & $2.9(3)$ & $3.1(3)$ & $3.0(4)$ & $2.7(3)$ \\
\hline 77 (7) & $4.2(4)$ & $3.6(3)$ & $3.3(4)$ & $2.6(3)$ \\
\hline $91(7)$ & $5.3(5)$ & $4.3(4)$ & $3.0(4)$ & $2.5(3)$ \\
\hline $105(7)$ & $5.2(5)$ & $4.6(4)$ & $2.8(3)$ & $2.1(3)$ \\
\hline $119(7)$ & $5.1(5)$ & $4.1(4)$ & $2.6(3)$ & $1.7(2)$ \\
\hline $133(7)$ & $3.9(4)$ & $3.5(3)$ & $2.2(3)$ & $1.2(2)$ \\
\hline $147(7)$ & $3.1(4)$ & $2.6(3)$ & $1.3(2)$ & 0.9 (1) \\
\hline $161(7)$ & $2.5(3)$ & $1.8(2)$ & $0.9(1)$ & $0.6(1)$ \\
\hline $175(7)$ & $1.9(2)$ & $1.2(2)$ & $0.6(1)$ & $0.46(9)$ \\
\hline $189(7)$ & $1.5(2)$ & 0.9 (1) & $0.31(6)$ & $0.24(6)$ \\
\hline $203(7)$ & $1.2(2)$ & $0.6(1)$ & $0.26(6)$ & $0.08(3)$ \\
\hline $217(7)$ & $0.8(1)$ & $0.39(7)$ & $0.11(4)$ & $0.04(2)$ \\
\hline $231(7)$ & 0.7 (1) & $0.25(6)$ & $0.04(2)$ & 0.021 (17) \\
\hline $245(7)$ & $0.45(9)$ & 0.07 (3) & $0.03(2)$ & $0.020(17)$ \\
\hline $259(7)$ & $0.32(7)$ & $0.05(3)$ & I & I \\
\hline $273(7)$ & $0.13(4)$ & I & / & / \\
\hline
\end{tabular}

Table A10. Double-differential cross sections of tritons with respect to the emission angle (columns) and kinetic energy (rows). The values in parentheses represent the uncertainties in the last digits.

\begin{tabular}{|c|c|c|c|c|}
\hline $\begin{array}{l}\mathrm{t} \\
\text { Energy } \\
(\mathrm{MeV})\end{array}$ & $\begin{array}{l}\theta_{\text {lab }}=11.4^{\circ} \\
\partial^{2} \sigma / \partial \Omega \partial E \\
\mathrm{mb} /(\operatorname{sr} \mathrm{MeV})\end{array}$ & $\begin{array}{l}\theta_{\text {lab }}=14.4^{\circ} \\
\partial^{2} \sigma / \partial \Omega \partial E \\
\mathrm{mb} /(\mathrm{sr} \mathrm{MeV})\end{array}$ & $\begin{array}{l}\theta_{\mathrm{lab}}=17.2^{\circ} \\
\partial^{2} \sigma / \partial \Omega \partial E \\
\mathrm{mb} /(\mathrm{sr} \mathrm{MeV})\end{array}$ & $\begin{array}{l}\theta_{\text {lab }}=19.4^{\circ} \\
\partial^{2} \sigma / \partial \Omega \partial E \\
\mathrm{mb} /(\operatorname{sr~MeV})\end{array}$ \\
\hline $10(10)$ & $0.64(8)$ & $0.49(6)$ & $0.30(4)$ & $0.26(4)$ \\
\hline $30(10)$ & $1.3(1)$ & $1.1(1)$ & 0.9 (1) & 0.7 (1) \\
\hline $50(10)$ & $1.6(1)$ & $1.0(1)$ & $0.89(9)$ & 0.7 (1) \\
\hline $70(10)$ & $2.0(2)$ & $1.2(1)$ & $0.8(1)$ & $0.66(9)$ \\
\hline $90(10)$ & $2.1(2)$ & $1.5(2)$ & 0.9 (1) & $0.8(1)$ \\
\hline $110(10)$ & $2.4(2)$ & $1.7(2)$ & $0.9(1)$ & $0.8(1)$ \\
\hline $130(10)$ & $2.5(2)$ & $1.7(2)$ & 0.7 (1) & $0.61(9)$ \\
\hline $150(10)$ & $2.3(2)$ & $1.6(2)$ & $0.56(9)$ & $0.48(9)$ \\
\hline $170(10)$ & $2.0(2)$ & $1.6(2)$ & $0.46(8)$ & $0.36(7)$ \\
\hline 190 (10) & $1.6(2)$ & $1.1(1)$ & $0.41(7)$ & $0.23(6)$ \\
\hline $210(10)$ & $1.4(2)$ & $0.67(8)$ & $0.27(6)$ & $0.17(5)$ \\
\hline $230(10)$ & $0.9(1)$ & $0.40(6)$ & $0.18(4)$ & $0.10(4)$ \\
\hline $250(10)$ & 0.7 (1) & $0.21(4)$ & 0.07 (2) & 0.023 (17) \\
\hline 270 (10) & $0.47(8)$ & $0.11(3)$ & $0.04(2)$ & 0.020 (17) \\
\hline 290 (10) & 0.37 (7) & 0.04 (2) & $0.015(12)$ & I \\
\hline 310 (10) & $0.15(4)$ & $0.03(2)$ & I & / \\
\hline 330 (10) & $0.05(2)$ & $0.015(12)$ & / & / \\
\hline $350(10)$ & $0.04(2)$ & I & / & / \\
\hline 370 (10) & $0.013(12)$ & / & / & / \\
\hline
\end{tabular}


Table A11. Cross sections of $Z>1$ fragments measured at different angles. The values in parentheses represent the uncertainties in the last digits.

\begin{tabular}{llllllll}
\hline$\theta_{\text {lab }}$ & ${ }^{4} \mathrm{He}$ & ${ }^{6} \mathrm{Li}$ & ${ }^{7} \mathrm{Li}$ & ${ }^{7} \mathrm{Be}$ & ${ }^{9} \mathrm{Be}$ & ${ }^{10} \mathrm{~B}$ & \\
$(\mathrm{Degree})$ & $\mathrm{d} \sigma / \mathrm{d} \Omega$ & $\mathrm{d} \sigma / \mathrm{d} \Omega$ & $\mathrm{d} \sigma / \mathrm{d} \Omega$ & $\mathrm{B}$ \\
$(\mathrm{b} / \mathrm{sr})$ & $(\mathrm{b} / \mathrm{sr})$ & $\begin{array}{l}\mathrm{d} \sigma / \mathrm{d} \Omega \\
(\mathrm{b} / \mathrm{sr})\end{array}$ & $\begin{array}{l}\mathrm{d} \sigma / \mathrm{d} \Omega \\
(\mathrm{b} / \mathrm{sr})\end{array}$ & $\begin{array}{l}\mathrm{d} \sigma / \mathrm{d} \Omega \\
(\mathrm{b} / \mathrm{sr})\end{array}$ & $\begin{array}{l}\mathrm{d} \sigma / \mathrm{d} \Omega \\
(\mathrm{b} / \mathrm{sr})\end{array}$ \\
\hline $2.2(0.4)$ & $11.3(1.1)$ & $0.88(19)$ & $0.94(20)$ & $0.81(20)$ & $0.59(16)$ & $1.62(32)$ & $1.90(35)$ \\
$2.9(0.4)$ & $11.2(1.1)$ & $0.93(20)$ & $0.75(17)$ & $0.80(20)$ & $0.47(14)$ & $1.01(23)$ & $1.74(33)$ \\
$3.5(0.4)$ & $9.8(1.0)$ & $0.86(19)$ & $0.74(17)$ & $0.79(20)$ & $0.46(14)$ & $1.02(25)$ & $1.40(31)$ \\
$4.1(0.4)$ & $9.27(98)$ & $0.60(14)$ & $0.59(15)$ & $0.45(13)$ & $0.33(11)$ & $/$ & $/$ \\
$4.9(0.4)$ & $6.84(74)$ & $0.46(11)$ & $0.56(14)$ & $0.48(14)$ & $0.28(11)$ & $0.57(17)$ & $0.79(19)$ \\
$7.6(1.5)$ & $4.42(39)$ & $0.371(43)$ & $0.446(58)$ & $0.312(39)$ & $0.147(24)$ & $0.288(41)$ & $0.390(54)$ \\
$8.2(1.5)$ & $4.04(35)$ & $0.295(36)$ & $0.366(43)$ & $0.225(30)$ & $/$ & $/$ & $/$ \\
$8.6(1.5)$ & $4.25(37)$ & $0.318(34)$ & $0.432(46)$ & $0.250(29)$ & $0.113(17)$ & $0.242(32)$ & $/$ \\
$11.1(1.5)$ & $2.76(24)$ & $0.178(24)$ & $0.224(31)$ & $0.129(21)$ & $0.046(12)$ & $0.080(17)$ & $0.087(18)$ \\
$12.1(1.5)$ & $2.30(19)$ & $0.129(16)$ & $0.166(20)$ & $0.096(13)$ & $0.036(7)$ & $0.048(9)$ & $0.048(9)$ \\
$12.8(1.5)$ & $1.83(16)$ & $0.107(14)$ & $0.112(15)$ & $0.073(11)$ & $0.021(5)$ & $0.030(6)$ & $0.025(6)$ \\
$14.4(1.5)$ & $1.38(12)$ & $0.071(9)$ & $0.078(10)$ & $0.043(6)$ & $0.009(3)$ & $0.014(3)$ & $0.009(2)$ \\
$15.2(1.5)$ & $1.08(10)$ & $0.061(12)$ & $0.059(12)$ & $0.040(9)$ & $/$ & $/$ & $/$ \\
$15.7(1.5)$ & $1.057(90)$ & $0.055(7)$ & $0.049(7)$ & $0.032(5)$ & $/$ & $/$ & $/$ \\
$16.4(1.5)$ & $1.003(88)$ & $0.051(8)$ & $0.052(9)$ & $0.029(6)$ & $/$ & $/$ & $/$ \\
$16.8(1.5)$ & $0.924(80)$ & $0.049(7)$ & $0.045(7)$ & $0.028(5)$ & $/$ & $/$ & $/$ \\
$17.2(1.5)$ & $0.783(66)$ & $0.038(5)$ & $0.031(4)$ & $0.021(3)$ & $/$ & $/$ & $/$ \\
$18.0(1.5)$ & $0.724(63)$ & $0.034(5)$ & $0.027(4)$ & $0.018(3)$ & $/$ & $/$ & $/$ \\
$19.0(1.5)$ & $0.681(65)$ & $0.034(8)$ & $0.028(7)$ & $0.020(6)$ & $/$ & $/$ & $/$ \\
$19.4(1.5)$ & $0.475(43)$ & $0.023(4)$ & $0.018(3)$ & $0.011(2)$ & $/$ & $/$ & $/$ \\
$20.6(1.5)$ & $0.487(44)$ & $0.022(4)$ & $0.020(4)$ & $0.009(2)$ & $/$ & $/$ & $/$ \\
$21.8(1.5)$ & $0.402(36)$ & $0.018(3)$ & $0.014(2)$ & $0.007(2)$ & $/$ & $/$ & $/$ \\
\hline
\end{tabular}

Table A12. Cross sections of $Z=1$ fragments measured at different angles. The values in parentheses represent the uncertainties in the last digits.

\begin{tabular}{llll}
\hline $\begin{array}{l}\theta_{\text {lab }} \\
(\text { Degree })\end{array}$ & $\begin{array}{l}p \\
\mathrm{~d} \sigma / \mathrm{d} \Omega \\
(\mathrm{b} / \mathrm{sr})\end{array}$ & $\begin{array}{l}d \\
\mathrm{~d} \sigma / \mathrm{d} \Omega \\
(\mathrm{b} / \mathrm{sr})\end{array}$ & $\begin{array}{l}t \\
\mathrm{~d} \sigma / \mathrm{d} \Omega \\
(\mathrm{b} / \mathrm{sr})\end{array}$ \\
\hline $11.4(1.5)$ & $1.02(11)$ & $0.639(70)$ & $0.450(46)$ \\
$14.4(1.5)$ & $0.915(88)$ & $0.520(52)$ & $0.290(32)$ \\
$15.7(1.5)$ & $0.732(74)$ & $0.416(44)$ & $0.193(23)$ \\
$17.2(1.5)$ & $0.646(64)$ & $0.387(51)$ & $0.146(21)$ \\
$19.4(1.5)$ & $0.483(51)$ & $0.312(36)$ & $0.119(19)$ \\
$20.6(1.5)$ & $0.472(51)$ & $0.272(31)$ & $0.064(8)$ \\
$21.8(1.5)$ & $0.418(48)$ & $0.252(31)$ & $0.062(8)$ \\
\hline
\end{tabular}

\section{Acknowledgments}

This work was supported by the Ion Beam Applications (IBA) company and by the European Space Agency (ESA) (tender AO6041). The authors wish to thank Dr V Greco (Dipartimento di Fisica e Astronomia, Università di Catania) for the useful discussions.

\section{References}

Agostinelli S et al (Geant4 Collaboration) 2003 Nucl. Instrum. Methods 506 250-303

Aichelin J 1991 Phys. Rep. 202 233-60 and references therein 
Allison J et al (Geant4 Collaboration) 2006 IEEE Trans. Nucl. Sci. 53 270-8

Amaldi U and Kraft G 2005 Rep. Prog. Phys. 68 1861-82

Battistoni G et al 2007 The FLUKA code: description and benchmarking AIP Conf. Proc. Proc. Hadronic Shower Simulation Workshop 2006; 8963149

Böhlen T T et al 2010 Phys. Med. Biol. 55 5833-47

Czudek J et al 1991 Phys. Rev. C 43 1248-54

Dayras R et al 1986 Nucl. Phys. A 460 299-323

Dementyev A V and Sobolevsky N M 1999 SHIELD universal Monte Carlo hadron transport code: scope and applications Radiat. Meas. 305537

Fassò A et al 2005 FLUKA: a multi-particle transport code Technical Report CERN-2005-10, INFN/TC 05/11, SL AC-R-773

Folger G, Ivanchenko V N and Wellisch J P 2004 Eur. Phys. J. A 21407

Glasow R et al 1990 Nucl. Part. Phys. 16 1089-94

Gudowska I et al 2004 Ion beam transport in tissue-like media using the Monte Carlo code SHIELD-HIT Phys. Med. Biol. 49 1933-58

Guet C et al 1983 Nucl. Phys. A 400 191c-220c

Hughes G et al 1997 MCNPXTM: the LAHETTM/MCNPTM code merger KEK Proc. 3rd Workshop on Simulating Accelerator Radiation Environments (SARE 3) (Tsukuba, Japan, 7-9 May) p 4451

Jacak B V et al 1987 Phys. Rev. C 351751

Koi T 2010 New native QMD code in Geant4 Proc. Joint Int. Conf. on Supercomputing in Nuclear Applications and Monte Carlo (Tokyo, Japan, 17-20 October 2010)

Krämer M and Durante M 2010 Eur. Phys. J. D 60 195-202

LANL 2002 MCNPX Users Manual Version 2.4.0

Niita K et al 1995 Phys. Rev. C 52 2660-35

Niita K et al 2006 PHITS: a particle and heavy ion transport code system Radiat. Meas. 41108090

Pruneau C et al 1989 Nucl. Phys. A 500 168-94

Raciti G et al 2008 Phys. Rev. Lett. 100192503

Schardt D, Elsässer T and Schulz-Ertner D 2010 Rev. Mod. Phys. 82 383-425

Sihver L and Mancusi D 2009 Radiat. Meas. 44 38-46

Tarasov L S et al 1998 Nucl. Phys. A 629 605-20

Tarasov O 2004 Nucl. Phys. A 734 536-40

Wada R et al 1989 Phys. Rev. C 39 497-515 